\title{
LOCAL DYNAMICS OF CONFORMAL VECTOR FIELDS
}

\author{
CHARLES FRANCES
}

\begin{abstract}
We study pseudo-Riemannian conformal vector fields in the neighborhood of a singularity. For Riemannian manifolds, we prove that if a conformal vector field vanishing at a point $x_{0}$ is not Killing for a metric in the conformal class, then a neighborhood of the singularity $x_{0}$ is conformally flat.
\end{abstract}

\section{INTRODUCTION}

A smooth vector field on a pseudo-Riemannian manifold $(M, g)$ is conformal whenever its local flow leaves invariant the conformal class $[g]:=\left\{e^{\sigma} g \mid \sigma \in\right.$ $\left.C^{\infty}(M)\right\}$. Otherwise stated, $X$ is a conformal vector field when there exists $\psi \in C^{\infty}(M)$ such that $\mathcal{L}_{X} g=\psi g$.

Locally, on a pseudo-Riemannian manifold of type $(p, q)$ and dimension $n=$ $p+q, n \geq 3$, the Lie algebra of conformal vector fields is finite dimensional, of dimension smaller or equal to $\frac{(n+2)(n+1)}{2}$. For every type $(p, q)$, there exists a compact, conformally flat manifold, for which this maximal dimension is attained, not only locally but also for the Lie algebra of global conformal vector fields. This manifold, called Einstein's universe $\operatorname{Ein}^{p, q}$, is obtained projectivizing in $\mathbf{R} \mathbf{P}^{n+1}$ the null cone of a type- $(p+1, q+1)$ quadratic form (see section 2.1). Any 1-parameter subgroup of $\mathrm{O}(p+1, q+1)$ acting on $\operatorname{Ein}^{p, q}$ defines a conformal vector field. Such fields on $\operatorname{Ein}^{p, q}$ will be called Möbius vector fields. They are a handy family of model fields, that one can study explicitely.

Studying conformal vector fields on a general manifold $(M, g)$ is a more difficult problem. One would like to understand completely the behavior of such fields around a singularity, and if possible to exhibit a complete family of normal forms. Though this problem has motivated quite a lot of works,

Date: May 28, 2018.

1991 Mathematics Subject Classification. 53A30, 53C50.

Key words and phrases. Conformal vector fields, pseudo-Riemannian structures. 
mainly in Riemannian $(p=0)$ or Lorentzian $(p=1)$ geometry (see, among other references [BCH], [Ca1], Ca2], [Fr1], KR1], [KR2], [KR3], [St]...), the local understanding of conformal vector fields is still not fully satisfactory.

A relevant notion, when studying conformal vector fields is that of essentiality. If $X$ is a conformal vector field on $(M, g)$, vanishing at $x_{0} \in M$, one says $X$ is inessential in the neighborhood of $x_{0}$ whenever there is an open set $U$ containing $x_{0}$, and a smooth function $\sigma: U \rightarrow \mathbf{R}$ such that $X$ is a Killing field for $e^{\sigma} g$ on $U$; this means $\mathcal{L}_{X}\left(e^{\sigma} g\right)=0$. In this case, the exponential map of $e^{\sigma} g$ allows to linearize $X$ near $x_{0}$ : indeed, it conjugates the action of the local flow $\left\{\phi_{X}^{t}\right\}$ generated by $X$, and that of the flow of the differential $\left\{D_{x_{0}} \phi_{X}^{t}\right\}$, on suitable neighborhoods of $x_{0}$ and $0 \in T_{x_{0}} M$ respectively. Hence, inessential conformal vector fields are easy to understand. Now, what can be said about essential vector fields, i.e those which do not preserve any metric in the conformal class $[g]$, on any neighborhood of the singularity $x_{0}$ ?

Our aim in this paper is to use the canonical Cartan connection associated to a pseudo-Riemannian conformal structure to study conformal vector fields. This is not really a new idea, since it already appeared, for instance, in [A] or Ca1, but we would like to sistemize it.

The upshot is that, as soon as we are dealing with manifolds of dimension at least three, the canonical Cartan connection associated to a conformal structure allows to define a distinguished class of curves: the conformal geodesics. This family is preserved by any local conformal transformation. Next, a base point $o$ being fixed once for all on the model space $\operatorname{Ein}^{p, q}$, it is possible to associate naturally to $X$ and the singularity $x_{0}$ a Möbius field $X_{h}$, vanishing at $o$ : this is the holonomy vector field of $X$ at $x_{0}$. This field $X_{h}$ generates a 1-parameter group $\left\{h^{t}\right\}:=\left\{\exp \left(t X_{h}\right)\right\} \subset \mathrm{O}(p+1, q+1)$, fixing $o$, and called the holonomy flow. A fundamental fact is that the action of the local flow $\left\{\phi_{X}^{t}\right\}$ on conformal geodesic segments emanating from $x_{0}$ is actually conjugated to the action of $\left\{h^{t}\right\}$ on the geodesic segments emanating from $o$. Unfortunately, unlike what is happening in the isometric case, one can not infer directly a local conjugacy between $X$ and $X_{h}$. This is basically because there are "too many" conformal geodesics for the "conformal exponential map" being a local diffeomorphism in a neighborhood of the origin. Yet, we will show that much of the local properties of $X$ around $x_{0}$ are encoded in its holonomy field $X_{h}$, and our aim is to develop this idea 
of a dictionnary between a conformal field and its holonomy, and to give evidences that there should be a positive answer to the following:

Question 1.1. In dimension $n \geq 3$, is a pseudo-Riemannian conformal vector field $X$ always locally conjugated in a neighborhood of a singularity $x_{0}$ to its holonomy vector field $X_{h}$ (in particular to a Möbius vector field)?

The formulation is deliberately vague concerning the regularity of the conjugacy, if it exists. We may have only a $C^{0}$-conjugacy between orbits of the local flows.

One of the main results of the article, is to get a complete description of Riemannian conformal vector fields around a singularity. In particular, we answer affirmatively to question 1.1 in this case (we will consider this question in Lorentzian signature in [FM2]). More precisely, we prove:

Theorem 1.2. Let $(M, g)$ be a smooth Riemannian manifold of dimension $n \geq 3$, endowed with a smooth conformal vector field $X$. We assume that $X$ vanishes at $x_{0} \in M$. Then:

(1) Either there is a neighborhood $U$ of $x_{0}$ on which $X$ is complete, and generates a flow which is relatively compact in Conf $(U)$. In this case $X$ is linearizable around $x_{0}$, and is inessential on $U$.

(2) If we are not in the previous case, there is a conformally flat neighborhood $U$ of $x_{0}$. The field $X$ is essential on each neighborhood of $x_{0}$.

In both cases, $X$ is smoothly conjugated in the neighborhood of $x_{0}$ to its holonomy field $X_{h}$.

This theorem is actually a particular case of theorem 7.1, which deals not only with single vector fields, but with any subalgebra of conformal vector fields.

If one adds a completeness assumption on the field $X$, theorem 1.2 reduces to a particular case of the so-called Obata-Ferrand's theorem (see [Ob], [F], Sch], Fr2]). This theorem states that a group of global conformal transformations on a Riemannian manifold $(M, g)$ is always inessential (i.e it acts isometrically for a metric in the conformal class $[g]$ ) except maybe if $(M,[g])$ is conformally equivalent to the standard sphere or the Euclidean space. The (independant) proofs of $[\mathrm{F}]$ and $[\mathrm{Sch}$ rely on the use of global conformal invariants, and thus do not seem to carry over into the study of 
conformal vector fields. In [A], D. Alekseevskii proposed a proof of theorem 1.2 in the case of complete vector fields, but some of his arguments turned out to be flawed. Nevertheless, Section 2 of $[\mathrm{A}$ develops ideas which are very similar to our notion of holonomy (see also [F3] for some complements about Aleksevskii's work).

1.1. Organisation of the paper. Sections 2 and 3 introduce some material about Einstein's universe, the interpretation of conformal structures of dimension $\geq 3$ as Cartan geometries, and the definition of conformal geodesics.

Then comes the heart of the paper, with the notion of holonomy of a vector field, introduced in section 4. We give a characterization of local inessentiality, as well as linearizability, in terms of holonomy (propositions 4.2 and 4.8).

The main difficulty in theorem 1.2 is that the vector fields we are considering are not assumed to be complete: all the orbits of the local flow $\left\{\phi_{X}^{t}\right\}$ (except that of $x_{0}$ ) could blow-up in finite time. It is thus crucial to exhibit conditions, for any type- $(p, q)$ manifold, ensuring that a conformal vector field $X$ admits "a lot" (actually a nonempty open set) of semi-complete orbits. By semi-complete, we mean defined on $]-\infty, 0]$ or $[0, \infty[$. This will be done in sections 5 and 6 .

When one is able to show that some orbits are semi-complete, then one can study asymptotic properties of those orbits, and using dynamical arguments, get geometrical informations such as, for instance, conformal flatness. This is the basic idea behind theorem 7.1, which is proved in the last section.

\section{Conformal structures and Cartan geometries}

2.1. Einstein's universe. In a lot of geometrical contexts, there exists among all possible structures, a distinguished object characterized by its great amount of symmetries. For type- $(p, q)$ conformal structures, this object is Einstein's universe $\operatorname{Ein}^{p, q}$, of which we give now a brief description (see [BCDGM] or [Fr3 for more details). In the whole paper, we assume that $p \leq q$ (in case $q>p$, one should switch the words "time" and "space" in all the statements).

Let us call $\mathbf{R}^{p+1, q+1}$ the space $\mathbf{R}^{p+q+2}$ endowed with the quadratic form:

$$
Q^{p+1, q+1}(x):=2 x_{0} x_{p+q+1}+\ldots+2 x_{p} x_{q+1}+\Sigma_{p+1}^{q} x_{i}^{2}
$$


The null cone of $Q^{p+1, q+1}$ is denoted $\mathcal{N}^{p+1, q+1}$. When restricted to $\mathcal{N}^{p+1, q+1}$, the form $Q^{p+1, q+1}$ yields a degenerate metric, the (1-dimensional) kernel of which is tangent to the cone. Thus, the projectivization $\mathbf{P}\left(\mathcal{N}^{p+1, q+1} \backslash\{0\}\right)$ is a smooth submanifold of $\mathbf{R} \mathbf{P}^{p+q+1}$, naturally endowed with a conformal class of type- $(p, q)$ metrics. One calls Einstein's universe of type $(p, q)$, denoted by $\operatorname{Ein}^{p, q}$, this compact manifold $\mathbf{P}\left(\mathcal{N}^{p+1, q+1} \backslash\{0\}\right)$ endowed with the conformal structure described above.

Notice that the space $\operatorname{Ein}^{0, q}$ is merely the sphere $\mathbf{S}^{q}$ endowed with the conformal class of the round metric $g_{\mathbf{S}^{q}}$. For $p \geq 1$, the product $\mathbf{S}^{p} \times \mathbf{S}^{q}$, endowed with the conformal class of the product metric $-g_{\mathbf{S}^{p}} \oplus g_{\mathbf{S}^{q}}$ is a double cover of de $\operatorname{Ein}^{p, q}$.

Let $\mathrm{O}(p+1, q+1)$ be the group of linear transformations preserving $Q^{p+1, q+1}$. Clearly, the natural action of $\mathrm{PO}(p+1, q+1)$ on $\operatorname{Ein}^{p, q}$ preserves the conformal class of $\operatorname{Ein}^{p, q}$, and actually $\mathrm{PO}(p+1, q+1)$ turns out to be the whole group of conformal transformations of $\operatorname{Ein}^{p, q}$ (this is Liouville's theorem, see [Fr3] or [S], and references in [KR4]).

In the whole paper, we will call $o$ the point of $\operatorname{Ein}^{p, q}$ corresponding to $\left[e_{0}\right]$. Its stabilizer $P \subset \mathrm{PO}(p+1, q+1)$ is a parabolic subgroup isomorphic to the semi-direct product $\left(\mathbf{R}_{+}^{*} \times \mathrm{O}(p, q)\right) \ltimes \mathbf{R}^{p, q}=\operatorname{Conf}\left(\mathbf{R}^{p, q}\right)$. From the conformal point of view, $\operatorname{Ein}^{p, q}$ is the homogeneous space $\mathrm{PO}(p+1, q+1) / P$.

Let $j: \mathbf{R}^{p, q} \rightarrow \operatorname{Ein}^{p, q}$ be the map given in projective coordinates on $\mathbf{R} \mathbf{P}^{p+1, q+1}$ by:

$$
j:\left(\begin{array}{c}
x_{1} \\
\vdots \\
x_{n}
\end{array}\right) \mapsto\left[\begin{array}{c}
-\frac{Q^{p, q}(x)}{2} \\
x_{1} \\
\vdots \\
x_{n} \\
1
\end{array}\right]
$$

The map $j$ is a conformal embedding from type- $(p, q)$ Minkowski's space $\mathbf{R}^{p, q}$ onto a dense open subset of $\operatorname{Ein}^{p, q}$. This map is the stereographic projection of pole $o$. The image $j\left(\mathbf{R}^{p, q}\right)$ is the complementary in $\operatorname{Ein}^{p, q}$ of the lightcone of vertex $o$, namely the set of all lightlike geodesics emanating from $o$. By a slight abuse of language, we will often identify in the sequel $\mathbf{R}^{p, q}$ and its image $j\left(\mathbf{R}^{p, q}\right)$, namely we will see Minkowski's space as an open subset of Einstein's universe. Notice that $j$ conjugates the action of $P$ on $j\left(\mathbf{R}^{p, q}\right)$ and the affine action of $P=\left(\mathbf{R}_{+}^{*} \times \mathrm{O}(p, q)\right) \ltimes \mathbf{R}^{p, q}$ on $\mathbf{R}^{p, q}$. 
The basepoint $o$ is not in $j\left(\mathbf{R}^{p, q}\right)$; it is located "at infinity". It is thus convenient to introduce a second conformal chart:

$$
j^{o}:\left(\begin{array}{c}
x_{1} \\
\vdots \\
x_{n}
\end{array}\right) \mapsto\left[\begin{array}{c}
1 \\
x_{1} \\
\vdots \\
x_{n} \\
-\frac{Q^{p, q}(x)}{2}
\end{array}\right]
$$

The map $j^{o}$ is a conformal diffeomorphism from $\mathbf{R}^{p, q}$ to an open subset of $\operatorname{Ein}^{p, q}$ containing $o$. We denote $\mathbf{R}_{o}^{p, q}$ this open set and we say that $j^{o}$ is the "chart at infinity".

Finally, let us notice that $\mathbf{R}_{o}^{p, q} \cap j\left(\mathbf{R}^{p, q}\right)$ is simply the image by $j$ of $\mathbf{R}^{p, q}$ with its null cone (with vertex 0 ) removed.

2.2. The Lie algebra $\mathfrak{o}(p+1, q+1)$. The Lie algebra $\mathfrak{o}(p+1, q+1)$ comprises all matrices $X$ of size $(p+q+2) \times(p+q+2)$ satisfying the indentity:

$$
X^{t} J_{p+1, q+1}+J_{p+1, q+1} X=0
$$

Here, $J_{p+1, q+1}$ is the matrix of the quadratic form $Q^{p+1, q+1}$ expressed in the base $\left(e_{0}, \ldots, e_{n+1}\right)$.

The algebra $\mathfrak{o}(p+1, q+1)$ writes as a sum $\mathfrak{n}^{-} \oplus \mathfrak{r} \oplus \mathfrak{n}^{+}$, where:

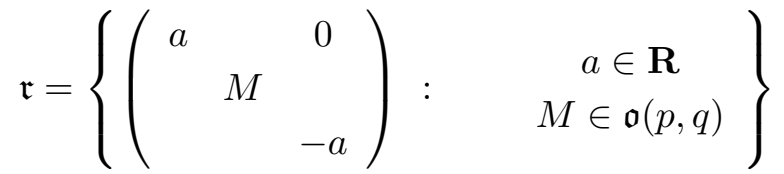

$$
\begin{aligned}
& \mathfrak{n}^{+}=\left\{\left(\begin{array}{ccc}
0 & -x^{t} \cdot J_{p, q} & 0 \\
& 0 & x \\
& & 0
\end{array}\right): \quad x \in \mathbf{R}^{p, q}\right\} \\
& \mathfrak{n}^{-}=\left\{\left(\begin{array}{ccc}
0 & & \\
x & 0 & \\
0 & -x^{t} . J_{p, q} & 0
\end{array}\right): \quad x \in \mathbf{R}^{p, q}\right\}
\end{aligned}
$$


In $\mathfrak{o}(p+1, q+1)$, one calls $\mathfrak{a}$ the algebra comprising the matrices:

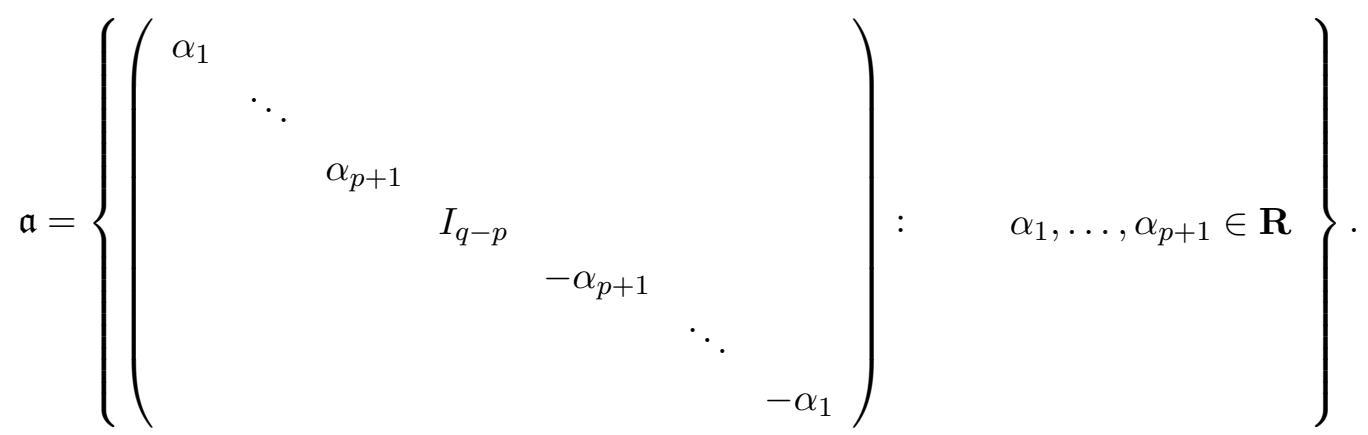

The closed subgroup of $P$ with Lie algebra $\mathfrak{a}$ is denoted by $A$. Let us call $\mathfrak{a}^{+}$the subset of $\mathfrak{a}$ corresponding to $\alpha_{1}, \ldots, \alpha_{p+1} \geq 0$, and $A^{+}:=\exp \left(\mathfrak{a}^{+}\right)$.

2.3. The parabolic subgroup $P$. The group $P$ is the stabilizer in $\mathrm{O}(p+$ $1, q+1)$ of the point $o=\left[e_{0}\right]$. As already seen, it is isomorphic to the semidirect product $\left(\mathbf{R}_{+}^{*} \times \mathrm{O}(p, q)\right) \ltimes \mathbf{R}^{p, q}$. Using the chart $j$, we will often see the elements of $P$ as affine transformations $A+T$, where $A \in \mathbf{R}_{+}^{*} \times \mathrm{O}(p, q)$ is the linear part, and $T \in \mathbf{R}^{p, q}$ is the translation factor.

As an element of $\mathrm{O}(p+1, q+1)$, a translation of vector $v \in \mathbf{R}^{p, q}$ writes:

$$
n^{+}(v):=\left(\begin{array}{ccc}
1 & -v^{t} \cdot J_{p, q} & -\frac{Q^{p, q}(v)}{2} \\
1 & v \\
& & 1
\end{array}\right) .
$$

The set of translations constitutes a group $N^{+}$, with Lie algebra $\mathfrak{n}^{+}$. The $\operatorname{map} n^{+}: \mathbf{R}^{p, q} \rightarrow N^{+}$is a group isomorphism.

Seen in $\mathrm{O}(p+1, q+1)$, an element $\lambda A \in \mathbf{R}_{+}^{*} \times \mathrm{O}(p, q)$ writes as:

$$
\left(\begin{array}{ccc}
\lambda & 0 & 0 \\
0 & A & 0 \\
0 & 0 & \frac{1}{\lambda}
\end{array}\right)
$$

2.4. The problem of equivalence. Let $G$ be a Lie group, $P \subset G$ a closed subgroup, and $\mathbf{X}=G / P$. A Cartan geometry modelled on $\mathbf{X}$ is the data of a triple $(M, B, \omega)$, where:

(1) $M$ is a manifold having the same dimenson as $\mathbf{X}$.

(2) $\pi: B \rightarrow M$ is a $P$-principal bundle over $M$.

(3) The form $\omega$ is a 1 -form on $B$ taking values in $\mathfrak{g}$, and satisfying:

(a) For every $b \in B, \omega_{b}: T_{b} B \rightarrow \mathfrak{g}$ is an isomorphism of vector spaces. 
(b) For every $X \in \mathfrak{g}$ and $b \in B, \omega_{b}\left(\frac{d}{d t \mid t=0} R_{\exp (t X)} \cdot b\right)=X$.

(c) For every $p \in P,\left(R_{p}\right)^{*} \omega=\left(\operatorname{Ad} p^{-1}\right) . \omega$.

Here, $R_{p}$ denotes the right action by $p$ on $B$, and exp is the the exponential map of $G$. A 1 -form $\omega$ as above is called a Cartan connexion on $B$.

One can see a Cartan geometry as a curved analogue of the flat model $\left(\mathbf{X}, G, \omega_{G}\right)$, where $\omega_{G}$ is the Maurer-Cartan form on $G$.

Let us now take the example of the homogeneous space $\mathbf{X}:=\operatorname{Ein}^{p, q}=$ $\mathrm{PO}(p+1, q+1) / P$, and of a Cartan geometry $(M, B, \omega)$ modelled on $\operatorname{Ein}^{p, q}$. Let us call $\pi: B \rightarrow M$ the bundle map. For every $x \in M$ and $b \in B$ above $x$, there exists a natural isomorphism:

$$
\iota_{b}: \mathfrak{g} / \mathfrak{p} \rightarrow T_{x} M
$$

defined by $\iota_{b}(\bar{\xi}):=D_{b} \pi\left(\omega_{b}^{-1}(\xi)\right)$, where $\xi$ represents the class $\bar{\xi} \in \mathfrak{g} / \mathfrak{p}$ in $\mathfrak{g}$. The isomorphism $\iota_{b}$ satisfies the equivariance relation:

$$
\iota_{b . p^{-1}}((\operatorname{Ad} p) \cdot \bar{\xi})=\iota_{b}(\bar{\xi}), \forall p \in P
$$

So, if $\mathcal{C}$ denotes the unique conformal class of type- $(p, q)$ scalar products which are $(\operatorname{Ad} P)$-invariant on $\mathfrak{g} / \mathfrak{p}, \iota_{b}(\mathcal{C})$ determines a type- $(p, q)$ conformal class on $T_{x} M$, which does not depend on the choice of $b \in B$ above $x$. In other words, a Cartan geometry $(M, B, \omega)$ modelled on $\operatorname{Ein}^{p, q}$ defines a conformal class $[g]$ of type- $(p, q)$ metrics on $M$.

If the bundle $B$ is fixed, there are a priori a lot of Cartan connections $\omega$ defining the class $[g]$ on $M$. The following theorem ensures that there is a suitable choice of normalizations which makes $\omega$ unique (this is the conformal analogue of the Levi-Civita connection). Precisely, see [Sh, ch 7], [Ko], one can state:

Theorem 2.1 (E. Cartan). Let $(M,[g])$ be a type- $(p, q)$ pseudo-Riemannian conformal structure, $p+q \geq 3$. Then there exists a unique normal Cartan geometry $(M, B, \omega)$, modelled on Ein ${ }^{p, q}$, defining the conformal structure $(M,[g])$ by the process described above. In particular, any local conformal diffeomorphism $\phi$ on $M$ lifts to a local automorphism of the bundle B (also denoted $\phi)$ preserving $\omega$.

In what follows, we will call the triple $(M, B, \omega)$ given by theorem 2.1 , the normal Cartan bundle defined by $(M,[g])$. 


\section{Conformal geodesics}

In all this section, $(M,[g])$ denotes a type- $(p, q)$ pseudo-Riemanniann conformal structure, of dimension $n \geq 3$. We see this conformal structure as a Cartan geometry modelled on $\operatorname{Ein}^{p, q}$, and we call $(M, B, \omega)$ the corresponding normal Cartan bundle. In the following, we will often call $G$ the Lie group $\mathrm{PO}(p+1, q+1)$.

3.1. Development of curves. Let $b \in B$ and $\hat{\alpha}: I \rightarrow B$ a $C^{1}$-curve such that $\hat{\alpha}\left(t_{0}\right)=b$. One defines the development of $\hat{\alpha}$ at $b$, denoted $\mathcal{D}_{b}(\hat{\alpha})$, as the unique curve $\hat{\beta}: I \rightarrow G$ satisfying $\hat{\beta}\left(t_{0}\right)=1_{G}$ and:

$$
\omega\left(\alpha^{\prime}\right)=\omega_{G}\left(\beta^{\prime}\right)
$$

Let now be $x \in M, b \in B$ in the fiber of $x$, and $\alpha: I \rightarrow M$ a $C^{1}$-curve such that $\alpha\left(t_{0}\right)=x$, then one defines its development at $x$ relatively to $b$ as follows:

$$
\mathcal{D}_{x}^{b}(\alpha)(t):=\pi_{G}\left(\mathcal{D}_{b}(\hat{\alpha})(t)\right)
$$

where $\pi_{G}$ stands for the projection from $G$ onto $\operatorname{Ein}^{p, q}=G / P$, and $\hat{\alpha}$ is a lift of $\alpha$ in $B$, such that $\hat{\alpha}\left(t_{0}\right)=b$. The definition of $\mathcal{D}_{x}^{b}(\alpha)$ does not depend on the lift $\hat{\alpha}$ because if $\lambda: I \rightarrow B$ and $p: I \rightarrow P$ are two $C^{1}$-curves, and if we call $\gamma(t)=\lambda(t) \cdot p(t)$, then:

$$
\omega\left(\gamma^{\prime}(t)\right)=(\operatorname{Ad} p(t))^{-1} \cdot \omega\left(\lambda^{\prime}(t)\right)+\omega_{G}\left(p^{\prime}(t)\right)
$$

Otherwise stated, $\mathcal{D}_{b}(\gamma)(t)=\mathcal{D}_{b}(\lambda)(t) \cdot p(t)$ and thus (see [Sh, p. 208]):

$$
\pi_{G}\left(\mathcal{D}_{b}(\gamma)(t)\right)=\pi_{G}\left(\mathcal{D}_{b}(\lambda)(t) \cdot p(t)\right)
$$

3.2. Conformal exponential map. The data of $Z$ in $\mathfrak{g}=\mathfrak{o}(p+1, q+1)$ defines naturally a vector field $\hat{Z}$ on $B$ by the relation $\omega(\hat{Z})=Z$. If $Z \in \mathfrak{g}$, we call $\psi_{Z}^{t}$ the local flow generated on $B$ by the field $\hat{Z}$. At each $b \in B$, we define $\mathcal{W}_{b} \subset \mathfrak{g}$ as the set of $Z$ such that $\psi_{Z}^{t}$ is defined for $t \in[0,1]$ at $b$. Then one defines the exponential map at $b$ :

$$
\exp (b,): \mathcal{W}_{b} \rightarrow B
$$

as:

$$
\exp (b, Z):=\psi_{\hat{Z}}^{1} \cdot b
$$

It is a standard fact that $\mathcal{W}_{b}$ is a neighborhood of 0 , and the map $\xi \mapsto$ $\exp (b, \xi)$ determines a diffeomorphism from an open set $\mathcal{V}_{b} \subset \mathcal{W}_{b}$ containing 0 onto a neighborhood of $b$ in $B$. 
One checks easily that if $x \in M, b \in B$ above $M, \xi \in \mathfrak{g}$ and $\alpha(s):=$ $\pi(\exp (b, s \xi))$, then the development $\beta(s):=\mathcal{D}_{x}^{b}(\alpha)(s)$ is given by $\beta(s)=$ $\pi_{G}(\exp (s \xi))$.

Let $f$ be a conformal transformation of $M$. Then $f_{*}(\hat{Z})=\hat{Z}$, and if $p \in$ $P,\left(R_{p}\right)_{*}(\hat{Z})=\hat{Z}_{p}$, where $Z_{p}:=\left(\operatorname{Ad} p^{-1}\right) . Z$. One infers the following important equivariance property:

$$
f(\exp (b, \xi)) \cdot p^{-1}=\exp \left(f(b) \cdot p^{-1},(\operatorname{Ad} p) \cdot \xi\right)
$$

3.3. Conformal geodesics. The Cartan connection and the exponential map allow to define a distinguished class of curves on $(M, g)$, namely the conformal geodesics. We won't try here to make the link between the definition given below, and previous ones as, for instance, that given in [F2] (see also [CSZ]). We will call parametrized conformal geodesics of $M$ through $x \in M$, any curve $s \mapsto \pi(\exp (b, s \xi))$, where $b \in B$ is in the fiber above $x$, $\xi \in($ Ad $P) \cdot \mathfrak{n}^{-}$, and $s$ takes values in an open interval $I$ containing 0 . One says the geodesic is timelike (resp. spacelike, resp. lightlike) if $\iota_{b}(\bar{\xi})$ is timelike (resp. spacelike, resp. lightlike), where $\bar{\xi}$ is the projection of $\xi$ on $\mathfrak{g} / \mathfrak{p}$. Because of the relation $\exp (b, s \xi)=\exp \left(\exp \left(b, s_{0}\right),\left(s-s_{0}\right) \xi\right)$, the tangent vector to a timelike (resp. spacelike, resp. lightlike) conformal geodesic is everywhere timelike (resp. spacelike, resp. lightlike). If $\alpha$ is a conformal geodesic defined on $I$, and $s_{0} \in I$, we will call $[\alpha]$ the set $\alpha([0, s])$, and say that $[\alpha]$ is a conformal geodesic segment (or shortly conformal segment) emanating from $x$.

Let us describe a little bit more precisely the conformal geodesics in the model $\operatorname{Ein}^{p, q}$. Following the formula given in section 2.2 for elements of $\mathfrak{n}^{-}$, a conformal geodesic writes in projective coordinates:

$$
s \mapsto p .\left[\begin{array}{c}
1 \\
s w_{1} \\
\vdots \\
s w_{n} \\
-s^{2} \frac{Q^{p, q}(w)}{2}
\end{array}\right]
$$

where $w:=\left(\begin{array}{c}w_{1} \\ \vdots \\ w_{n}\end{array}\right) \in \mathbf{R}^{p, q}$ and $p \in P$. 
The geodesic is timelike (resp. spacelike) if and only if $w$ is timelike (resp. spacelike). In this case, it writes $s \mapsto j\left(p \cdot\left(-\frac{1}{s} \frac{2 w}{Q^{p, q}(w)}\right)\right)$. Thus, any timelike (resp. spacelike) geodesic is of the form:

$$
s \mapsto j\left(\frac{1}{s} v+v_{0}\right)
$$

where $v \in \mathbf{R}^{p, q}$ is timelike (resp. spacelike), and $v_{0} \in \mathbf{R}^{p, q}$. We see in particular that in the chart $j$, timelike or spacelike geodesic segments emanating from $o$ are half lines.

In the chart at infinity $j^{o}$, a timelike (resp. spacelike) geodesic writes:

$$
s \mapsto \frac{2 s\left(v+s v_{0}\right)}{Q^{p, q}\left(v+s v_{0}\right)}
$$

Let us focus now on lightlike geodesics. They write:

$$
s \mapsto p \cdot\left[\begin{array}{c}
1 \\
s w_{1} \\
\vdots \\
s w_{n} \\
0
\end{array}\right]
$$

From the matrices given in section 2.3 for elements $p \in P$, it is easy to check that the lightlike geodesic segments emanating from $o$ and contained in $R_{o}^{p, q}$ read in the chart $j^{0}$ as lightlike line segments emanating from 0 . Their parametrization is of the form:

$$
s \mapsto\left[\begin{array}{c}
1 \\
\tau(s) w_{1} \\
\vdots \\
\tau(s) w_{n} \\
0
\end{array}\right]
$$

where $s \mapsto \tau(s)$ is an homographic transformation.

3.4. Some auxiliary metrics. To compare a curve and its development, we will introduce Riemannian metrics on $G$ and $B$ as follows. On $\mathbf{R}^{p, q}$, we denote $\langle x, x\rangle=x_{1}^{2}+\ldots+x_{n}^{2}$ the standard Euclidean product, and we call $\|$.$\| the norm it defines. We pull back this scalar product on \mathbf{R}_{o}^{p, q}$ by the map $\left(j^{o}\right)^{-1}$, what endows $\mathbf{R}_{o}^{p, q}$ with a flat Riemannian metric $\rho^{o}$. If 
$v:=\left(v_{1}, \ldots, v_{n}\right)^{t}$ (the transpose of $\left.\left(v_{1}, \ldots, v_{n}\right)\right)$, we set:

$$
n^{-}(v):=\left(\begin{array}{cccc}
1 & & \\
-J_{p, q} \cdot v^{t} & 1 & \\
-\frac{Q^{p, q}}{2} & v & 1
\end{array}\right)
$$

The morhism $n^{-}: \mathbf{R}^{n} \rightarrow N^{-}$is an isomorphism and the relation $j^{o}(v+w)=$ $n^{-}(v) \cdot j^{o}(w)$ shows that $N^{-}$acts simply transitively on $\mathbf{R}_{o}^{p, q}$, and the metric $\rho^{o}$ is $N^{-}$-invariant. Hence, it induces a left-invariant metric $\rho^{-}$on $N^{-}$. We denote $<,>_{\mathfrak{n}^{-}}$the scalar product induced by this metric on $\mathfrak{n}^{-}$, and $\|\cdot\|_{\mathfrak{n}^{-}}$ the associated norm.

In all the paper, if $r>0$, we will call $\mathcal{B}(0, r)$ (resp. $\mathcal{S}(0, r))$ the open ball (resp. the sphere) centered at 0 and of radius $r$ in $\mathfrak{n}^{-}$for the norm $\|.\|_{\mathfrak{n}^{-}}$. The ball $B(o, r)$ of center $o$ and radius $r$ for the metric $\rho^{o}$ is just $\pi_{G}(\exp (\mathcal{B}(0, r)))$.

Let us choose a scalar product $<,>_{\mathfrak{g}}$ on $\mathfrak{g}$, inducing $<,>_{\mathfrak{n}^{-}}$on $\mathfrak{n}^{-}$. The product $<,>_{\mathfrak{g}}$ allows to define a left-invariant Riemannian metric $\rho^{G}$ on $G$. Similarly, we define a Riemannian metric $\rho^{B}$ on $B$ by the formula:

$$
\rho_{b}^{B}(u, v):=<\omega_{b}(u), \omega_{b}(v)>_{\mathfrak{g}}, \quad b \in B, \quad u, v \in T_{b} B
$$

In what follows, if $\beta$ is a $C^{1}$-curve in $\mathbf{R}_{o}^{p, q}$, we will denote $L^{o}(\beta)$ its length with respect to the metric $\rho^{o}$.

One checks immediately that if $\alpha$ is a $C^{1}$-curve through $b \in B$, and if $\beta:=\mathcal{D}_{b}(\alpha)$, then the length of $\alpha$ with respect to $\rho^{B}$ is the length of $\beta$ with respect to $\rho^{G}$. Also, if $\beta$ is a $C^{1}$-curve of $N^{-}$through $1_{G}$, then its length relatively to $\rho^{G}$ is $L^{o}\left(\pi_{G}(\beta)\right)$, since by construction, the restriction of $\rho^{G}$ to $N^{-}$is $\rho^{-}$.

3.5. Degeneration properties. A key idea of the paper will be to recover the dynamics of a sequence of local conformal transformations thanks to their action on the geodesic segments. For this, we will have to understand how a sequence of geodesic segments can degenerate. This will be done understanding the link between a sequence of geodesic segments, and the sequence of its developments. One now proves some lemmas in this direction, mostely inspired by [Fr2].

We first show that the curves through $x$ in $M$, the development of which are "short" curves in $\mathbf{R}_{o}^{p, q}$, are themselves short. 
Lemma 3.1. Let $x \in M$, and $b \in B$ in the fiber of $x$. For any neighborhood $U$ of $x$, there is a real $r_{U}$ such that if $\left.\alpha:\right] a, b\left[\rightarrow M\right.$ is a $C^{1}$-curve through $x$, and if $\mathcal{D}_{x}^{b}(\alpha)$ is included in $\mathbf{R}_{o}^{p, q}$ with $L^{o}\left(\mathcal{D}_{x}^{b}(\alpha)\right) \leq r_{U}$, then the curve $\alpha$ is included in $U$.

Proof: let us denote by $B(b, R)$ the ball of center $b$ and radius $R$ for the metric $\rho^{B}$. We first choose $r_{U}$ such that $\pi\left(B\left(b, r_{U}\right)\right) \subset U$. Since $\mathcal{D}_{x}^{b}(\alpha)$ is included in $\mathbf{R}_{o}^{p, q}$, there exists $\left.p:\right] a, b\left[\rightarrow P\right.$, such that $\beta(t):=\mathcal{D}_{b}(\alpha)(t) \cdot p(t)$ is a curve of $N^{-}$through $1_{G}$. The length of $\gamma(t):=\alpha(t) \cdot p(t)$ with respect to $\rho^{B}$ is then equal to the length $l$ of $\beta$ with respect to $\rho^{G}$. But $l$ is nothing else than $L^{o}\left(\mathcal{D}_{x}^{b}(\alpha)\right)$, as mentioned in the previous paragraph. We conclude that $\gamma$ is included in $B\left(b, r_{U}\right)$, and since $\gamma$ projects on $\alpha$, we get that $\alpha$ is included in $U . \diamond$

We now prove:

Proposition 3.2. Let $R>0$ and $[\alpha]$ a geodesic segment emanating from $o$ and contained in $B(o, R)$, then:

$$
L^{o}([\alpha]) \leq 8 n R
$$

Proof: we work in the chart at infinity $j^{\circ}$, and we consider a geodesic segment $[\alpha]$ emanating from $o$, and included in $B(0, R)$ (the Euclidean ball of center 0 and radius $R$ ). If it is a lightlike geodesic segment, then as it was observed in section 3.3 , it is a line segment emanating from 0 and included in $B(0, R)$. Its length $L^{o}$ is then at most $R$ and the proposition holds clearly in this case.

Now, if $[\alpha]$ is timelike or spacelike, we know from formula (5) that:

$$
\alpha(s)=\frac{2 s\left(v+s v_{0}\right)}{Q^{p, q}\left(v+s v_{0}\right)}, s \in\left[0, s_{0}\right]
$$

Thus, $\alpha(s):=\left(\alpha_{1}(s), \ldots, \alpha_{n}(s)\right)=\left(\frac{P_{1}(s)}{Q_{1}(s)}, \ldots, \frac{P_{n}(s)}{Q_{n}(s)}\right)$, where $P_{1}, \ldots, P_{n}$, $Q_{1}, \ldots, Q_{n}$ are polynomials of degree at most 2 . We infer that for $i=$ $1, \ldots, n$, the derivative $\alpha_{i}^{\prime}(s)$ vanishes at most three times on $\left[0, s_{0}\right]$. Thus, the curve $\alpha_{i}:\left[0, s_{0}\right] \rightarrow[-R, R]$ has length at most $8 R$. Indeed, if $\beta$ : $\left[0, s_{0}\right] \rightarrow[-R, R]$ is a $C^{1}$-curve the derivative of which vanishes at most $m$ times, the length of this curve is at most $2(m+1) R$. We get finally the inequality:

$$
\int_{0}^{s_{0}} \sqrt{\left(\alpha_{1}^{\prime}(s)\right)^{2}+\ldots+\left(\alpha_{n}^{\prime}(s)\right)^{2}} d s \leq \int_{0}^{s_{0}}\left(\left|\alpha_{1}^{\prime}(s)\right|+\ldots+\left|\alpha_{n}^{\prime}(s)\right|\right) d s \leq 8 n R
$$


This yields the proposition. $\diamond$

Corollary 3.3. Let $x_{0} \in M, b_{0} \in B$ in the fiber of $x_{0}$. If $\left[\alpha_{k}\right]$ is a sequence of conformal geodesic segments emanating from $x_{0}$, and if $\mathcal{D}_{x_{0}}^{b_{0}}\left(\left[\alpha_{k}\right]\right)$ tends to $o$, then $\left[\alpha_{k}\right] \rightarrow x_{0}$.

Proof: for every neighborhood $U$ of $x_{0}$, the previous proposition yields an integer $K$ such that if $k \geq K$, then $L^{o}\left(\left[\alpha_{k}\right]\right)<r_{U}$, where $r_{U}$ is given by lemma 3.1. This same lemma then says that $\left[\alpha_{k}\right] \subset U$ for $k \geq K$. $\diamond$

\section{Holonomy, Linearizability and ESSEnTIALITy}

In all this section, we consider $(M, g)$ a type- $(p, q)$, smooth pseudo-Riemannian manifold , $p+q \geq 3$. The associated normal Cartan bundle is denoted by $(M, B, \omega)$. We assume there exists a smooth conformal vector field $X$ on $M$, having a singularity $x_{0} \in M$. We are going to explain how to associate naturally to $X$ a Möbius field $X_{h}$ : its holonomy at $x_{0}$. Then, we will begin to establish a dictionnary between the properties of $X$ in a neighborhood of $x_{0}$ and those of $X_{h}$ in a neighborhood of $o$, begining with two issues: linearizability and essentiality.

\subsection{Holonomy morphism, holonomy vector field, and holonomy} algebra. Let $\mathfrak{I}_{x_{0}}$ be the Lie algebra of conformal vector fields of $M$ vanishing at $x_{0}$. Let $X$ be a vector field of $\mathfrak{I}_{x_{0}}$. The local flow $\left\{\phi_{X}^{t}\right\}$ lifts to $B$, yielding a vector field on $B$, still denoted $X$, satisfying $\mathcal{L}_{X} \omega=0$ (here, $\mathcal{L}_{X}$ is the Lie derivative with respect to $X$ ). Thus, for each $b \in B$, we get a linear monomorphism $s_{b}: \mathfrak{I}_{x_{0}} \rightarrow \mathfrak{g}$ defined by:

$$
s_{b}(X)=\omega_{b}(X(b)) .
$$

Now, if $X, Y \in \mathfrak{I}_{x_{0}}$, we have the following formula [BFM, Lemma 2.1]:

$$
\kappa_{b}\left(\overline{s_{b}(X)}, \overline{s_{b}(Y)}\right)=s_{b}([X, Y])+\left[s_{b}(X), s_{b}(Y)\right],
$$

where $\kappa: B \rightarrow \operatorname{Hom}\left(\Lambda^{2}(\mathfrak{g} / \mathfrak{p}), \mathfrak{g}\right)$ is the curvature function of $(M, B, \omega)$ (see [Sh, Definition 3.22]), and $\overline{s_{b}(X)}, \overline{s_{b}(Y)}$ are the projections on $\mathfrak{g} / \mathfrak{p}$ of $s_{b}(X)$ and $s_{b}(Y)$. We thus see that as soon as $b_{0}$ is a point in the fiber of $x_{0},-s_{b_{0}}: \mathfrak{I}_{x_{0}} \rightarrow \mathfrak{p}$ is an embedding of Lie algebras, called the holonomy morphism at $b_{0}$. The image of $-s_{b_{0}}$ is into $\mathfrak{p}$, because $X$ vanishes at $x_{0}$ and its lift is then tangent to the fiber of $x_{0}$. The element $s_{b_{0}}(X) \in \mathfrak{p}$ defines a right-invariant vector field $X_{h}$ on $G=\mathrm{O}(p+1, q+1)$ : it will be called the 
holonomy field of $X$ at $x_{0}$ (relatively to $b_{0}$ ). The holonomy vector field is a conformal vector field of $\operatorname{Ein}^{p, q}$, hence a Möbius field, vanishing at $o$. The 1-parameter group $\left\{h^{t}\right\}$ of $P$ defined by $h^{t}:=\exp \left(t s_{b_{0}}(X)\right)$ will be called the holonomy flow of $X$ at $x_{0}$ (relatively to $b_{0}$ ). Let $\left\{\phi_{X}^{t}\right\}$ be the local flow defined by $X$, lifted to $B$. Then for every $t \in \mathbf{R}$, we have:

$$
\phi_{X}^{t} \cdot b_{0} \cdot h^{-t}=b_{0}
$$

Using equation (2), it is easy to check that if $\alpha: I \rightarrow M$ is a $C^{1}$-curve through $x_{0}$, and if the local flow $\left\{\phi_{X}^{t}\right\}$ is defined at each point of $\alpha$ for $t \in[0, \delta]$, then the following equivariance relation holds:

$$
\mathcal{D}_{x_{0}}^{b_{0}}\left(\phi_{X}^{t} \cdot \alpha\right)(s)=h^{t} \cdot \mathcal{D}_{x_{0}}^{b_{0}}(\alpha)(s), \text { for every } s \in I \text { and } t \in[0, \delta] .
$$

Also, read into the chart $j$, the flow $\left\{h^{t}\right\}$ is affine on $\mathbf{R}^{p, q}$, thus can be written $A^{t}+T_{t}$, where $A^{t} \in \mathbf{R}_{+}^{*} \times \mathrm{O}(p, q)$ is the linear part, and $T_{t} \in \mathbf{R}^{p, q}$ the translation part. The relation:

$$
D_{x_{0}} \phi_{X}^{t}\left(\iota_{b}(\xi)\right)=\iota_{b}\left(\left(\operatorname{Ad} h^{t}\right) \cdot \xi\right)
$$

identifies the linear part $A^{t}$ with the differential $D_{x_{0}} \phi_{X}^{t}$ read in an orthonormal frame of $T_{x_{0}} M$.

Finally, if we are considering $\mathfrak{h} \subset \mathfrak{I}_{x_{0}}$ a subalgebra, it is also possible to define its holonomy algebra $\mathfrak{h}_{h}:=s_{b_{0}}(\mathfrak{h})$. Its holonomy group $H_{h} \subset P$ is the connected subgroup of $P$ having $\mathfrak{h}_{h}$ as Lie algebra.

The definition of the holonomy group (and of the holonomy algebra) of $\mathfrak{h}$ at $x_{0}$ depends on the choice of $b_{0}$ in the fiber of $x_{0}$. If $b_{0}$ is replaced by $b_{0} . p$, with $p \in P$, then $H_{h}$ (resp. $\mathfrak{h}_{h}$ ) is changed into $p . H_{h} \cdot p^{-1}$ (resp. into $($ Ad $\left.p) \cdot \mathfrak{h}_{h}\right)$. The holonomy of $\mathfrak{h}$ at $x_{0}$ is thus well defined up to conjugacy in $P$. By a slight abuse of language, when we will speak about the holonomy of $\mathfrak{h}$ at $x_{0}$, we will mean a representative of the conjugacy class of all possible holonomies at $x_{0}$.

Remark 4.1. In case $\mathfrak{h}$ is a Lie algebra of conformal vector fields on a neighborhood $V$ of o in Ein ${ }^{p, q}$, then we merely have $\mathfrak{h}=\mathfrak{h}_{h}$. This follows from the fact that the normal Cartan bundle is the inverse image of $V$ in $\left(\right.$ Ein $\left.^{p, q}, O(p+1, q+1), \omega_{G}\right)$, and from Liouville's theorem.

4.2. Holonomy and linearizability. The property for $X$ to be linearizable in a neighborhood of $x_{0}$ can be seen very easily on its holonomy flow. This is the content of the following proposition. 
Proposition 4.2. The field $X$ is linearizable in a neighborhood of $x_{0}$ if and only if its holonomy flow $\left\{h^{t}\right\} \subset \operatorname{Conf}\left(\mathbf{R}^{p, q}\right)$ has a fixed point on $\mathbf{R}^{p, q}$. In this case, there is a $C^{\infty}$-diffeomorphism from a neighborhood of $x_{0}$ onto a neighborhood of o which conjugates $X$ and $X_{h}$.

Proof: we begin with the easiest part of the proposition. Let us assume that $\left\{h^{t}\right\}$ fixes a point of $\mathbf{R}^{p, q}$. Considering $b_{0} . p$ instead of $b_{0}$ for a suitable $p \in P$ (i.e conjugating $\left\{h^{t}\right\}$ by $p$ ), we can assume that this fixed point is 0 , namely $\left\{h^{t}\right\} \subset \mathbf{R}_{+}^{*} \times \mathrm{O}(p, q)$. We then pick $r>0$ small enough so that $\xi \mapsto \pi\left(\exp \left(b_{0}, \xi\right)\right)$ is a diffeomorphism, denoted $\psi$, from $\mathcal{B}(0, r)$ onto a neighborhood $U$ of $x_{0}$, and $\xi \mapsto \pi_{G}(\exp (\xi))$ is a diffeomorphism, denoted $\varphi$, from $\mathcal{B}(0, r)$ onto a neighborhood $V$ of $o$. For $t$ near 0 , and $\xi \in \mathcal{B}(0, r)$, the relation:

$$
\phi_{X}^{t} \cdot \exp \left(b_{0}, \xi\right) \cdot h^{-t}=\exp \left(b_{0},\left(\operatorname{Ad} h^{t}\right) \cdot \xi\right)
$$

just writes $\phi_{X}^{t} \circ \psi=\psi \circ\left(\operatorname{Ad} h^{t}\right)$, and is available on $\mathcal{B}(0, r)$. Thus, $\psi$ conjugates the local flow of $X$ on $U$ to the linear flow $\left(\operatorname{Ad} h^{t}\right)$ on $\mathcal{B}(0, r)$. Moreover, $\psi \circ \varphi^{-1}$ is a smooth diffeomorphism from $U$ onto $V$ which conjugates $X$ and $X_{h}$.

We are now going to prove the converse statement, namely that if $\left\{h^{t}\right\}$ does not fix a point in $\mathbf{R}^{p, q}$, then $X$ is not linearizable around $x_{0}$. In the chart $j,\left\{h^{t}\right\}$ is a flow of affine conformal transformations of $\mathbf{R}^{p, q}$, that we denote $h^{t}=e^{\lambda t} A^{t}+T_{t}$. Here $\left\{A^{t}\right\}$ is a 1-parameter subgroup of $\mathrm{O}(p, q)$, and $\lambda \in \mathbf{R}$.

Let us begin with a first basic remark. If $h=e^{\lambda} A+T$ is the time 1 of the flow $\left\{h^{t}\right\}$, and if $h$ has a fixed point $z_{0}$ on $\mathbf{R}^{p, q}$, then $\left\{h^{t}\right\}$ has also a fixed point on $\mathbf{R}^{p, q}$. Indeed, the orbit $h^{t} . z_{0}$ is compact. Its affine convex hull is compact as well. The flow $\left\{h^{t}\right\}$ is affine and leaves invariant a compact convex set: it must have a fixed point. We will thus assume in the following that $h=e^{\lambda} A+T$ does not fix any point on $\mathbf{R}^{p, q}$.

If $u \in \mathbf{R}^{p, q}$ is a nonzero lightlike vector, we can associate to $u$ a lightlike geodesic $\beta_{u}$ through $o$, given by the parametrization:

$$
\beta_{u}(s):=j^{o}(s u)
$$

We see $e^{\lambda} A$, and the translation of vector $T$, as elements of $P$ acting on $\operatorname{Ein}^{p, q}$. Then we can state:

Lemma 4.3. Let $u \in \mathbf{R}^{p, q}$ be a lightlike vector such that $e^{\lambda} A . u=e^{2 \lambda} u$. Then: 
(1) For every $s \in \mathbf{R}, e^{\lambda} A \cdot \beta_{u}(s)=\beta_{u}(s)$.

(2) Let $\langle,\rangle_{p, q}$ be the bilinear form associated to $Q^{p, q}$. If $s \neq \frac{1}{\langle T, u\rangle_{p, q}}$, then the translation of vector $T$ maps $\beta_{u}(s)$ to $\beta_{u}\left(\frac{s}{1-s\langle T, u\rangle_{p, q}}\right)$

Proof: It is a simple computation from the matrix expressions given in section 2.3. $\diamond$

The second step is to show:

Lemma 4.4. If $h$ does not fix any point of $\mathbf{R}^{p, q}$, there exists a lightlike vector $u \in \mathbf{R}^{p, q}$ satisfying $e^{\lambda} A . u=e^{2 \lambda} u$, and $\langle T, u\rangle_{p, q}=-1$.

Proof: we begin with a remark. Assume that $A$ preserves a splitting $\mathbf{R}^{p, q}=F \oplus H$, and assume that 1 is not an eigenvalue for the restriction of $e^{\lambda} A$ to $H$. Then, if $T_{H}$ is the component of $T$ on $H$, there exists $\tau \in H$ such that $\left(e^{\lambda} A-I d\right) \cdot \tau=T_{H}$. Then the translation part of the affine map $(I d+\tau) \circ h \circ(I d+\tau)^{-1}$ does not have any component along $H$. In other words, conjugating $h$ into $P$, we can assume that $T \in F$.

Let us perform a real Jordan decomposition of $A$, into the algebraic group $\mathrm{O}(p, q): A=A_{s} A_{e} A_{u}$, where $A_{s}, A_{e}$ and $A_{u}$ are all in $\mathrm{O}(p, q), A_{s}$ is Rsemisimple, $A_{e}$ is elliptic, namely $\mathbf{C}$-semisimple with all its eigenvalues of modulus one, and $A_{u}$ is unipotent. Moreover, the elements $A_{s}, A_{e}$ and $A_{u}$ are pairwise commuting. Let $F:=\operatorname{Ker}\left(A_{e}-I d\right)$. Because the group generated by $A_{e}$ is relatively compact in $\mathrm{O}(p, q), A_{e}$ preserves an orthogonal splitting $F^{\prime} \oplus H^{\prime}$, where $F^{\prime}$ has dimension $p$ and $Q_{\mid F^{\prime}}^{p, q}$ is Riemannian, and $H^{\prime}$ has dimension $q$ and $-Q_{\mid H^{\prime}}^{p, q}$ is Riemannian. As a consequence, $F$ is nondegenerate, of type $\left(p^{\prime}, q^{\prime}\right)$. We denote by $H$ the orthogonal of $F$, relatively to $\langle,\rangle_{p, q}$. Then $A_{e}$ preserves the splitting $\mathbf{R}^{p, q}=F \oplus H$. Observe that because $A_{s}$ and $A_{u}$ are commuting with $A_{e}, A$ also preserves the splitting $\mathbf{R}^{p, q}=F \oplus H$, and 1 is not an eigenvalue for the restriction of $e^{\lambda} A$ to $H$. By the previous remark, we can assume $T \in F$.

- Let us first handle the case where $\lambda=0$. The transformation $A_{\mid F}$ must admit 1 as an eigenvalue, otherwise $h$ would fix a point in $\mathbf{R}^{p, q}$ by the remark made at the begining of the proof. Let us call $F_{1} \subset F$ the eigenspace associated to 1 . Because $A_{\mid F}$ is in $\mathrm{O}\left(p^{\prime}, q^{\prime}\right)$, it is not hard to check that $\operatorname{Ker}(A-I d)_{\mid F}$ is the orthogonal of $\operatorname{Im}(A-I d)_{\mid F}$ in $F$. On the other hand, $T \notin \operatorname{Im}(A-I d)_{\mid F}$, otherwise $h$ would have a fixed point in $\mathbf{R}^{p, q}$. We infer 
that there exists $u \in \operatorname{Ker}(A-I d)_{\mid F}$ such that $\langle T, u\rangle_{p, q} \neq 0$. Rescaling $u$ if necessary, the proposition follows in this case.

- We now assume that $\lambda \neq 0$. In this case, $\left(A_{s}\right)_{\mid F}$ must admit $e^{-\lambda}$ as an eigenvalue, otherwise $h$ would have a fixed point, by the remark made at the begining of the proof. Let $F_{1}$ be the associated eigenspace of $e^{\lambda}\left(A_{s}\right)_{\mid F}$. Since $\left(A_{s}\right)_{\mid F}$ is $\mathbf{R}$-semisimple, we can write $F=F_{1} \oplus F_{2} \oplus \ldots \oplus F_{m}$, where each $F_{j}$ is an eigenspace for $\left(A_{s}\right)_{\mid F}$. Because $\left(A_{u}\right)_{\mid F}$ commutes with $\left(A_{s}\right)_{\mid F}$, this splitting is preserved by $\left(A_{u}\right)_{\mid F}$, hence by $e^{\lambda} A_{\mid F}$. By the remark made at the begining of the proof, we can assume that $T \in F_{1}$. Let us observe that because $A_{\mid F} \in \mathrm{O}\left(p^{\prime}, q^{\prime}\right)$, $e^{\lambda}$ must also be an eigenvalue of $A_{\mid F}$, and we will assume that the associated eigenspace is $F_{2}$. The sum $F_{1} \oplus F_{2}$ is then nondegenerate of type $\left(p^{\prime \prime}, p^{\prime \prime}\right)$, and the spaces $F_{1}, F_{2}$ are both totally degenerate (namely, $\langle,\rangle_{p, q}$ restricts to 0 on them). Because $\left(A_{u}\right)_{\mid F_{1} \oplus F_{2}}$ is in $\mathrm{O}\left(p^{\prime \prime}, p^{\prime \prime}\right)$, the spaces $\operatorname{Im}\left(A_{u}-I d\right)_{\mid F_{1} \oplus F_{2}}$ and $\operatorname{Ker}\left(A_{u}-I d\right)_{\mid F_{1} \oplus F_{2}}$ are orthogonal. Since $F_{1}$ and $F_{2}$ are left invariant by $A_{u}$, one has:

$$
\operatorname{Im}\left(A_{u}-I d\right)_{\mid F_{1} \oplus F_{2}}=\operatorname{Im}\left(A_{u}-I d\right)_{\mid F_{1}} \oplus \operatorname{Im}\left(A_{u}-I d\right)_{\mid F_{2}}
$$

Because $h$ does not fix any point in $\mathbf{R}^{p, q}, T \notin \operatorname{Im}\left(e^{\lambda} A-I d\right)$, and because $T \in F_{1}$, we actually get $T \notin \operatorname{Im}\left(e^{\lambda} A-I d\right)_{\mid F_{1}}$. On the other hand $\left(A_{u}\right)_{\mid F_{1}}=$ $e^{\lambda} A_{\mid F_{1}}$, so that $T \notin \operatorname{Im}\left(A_{u}-I d\right)_{\mid F_{1}}$, and finally $T \notin \operatorname{Im}\left(A_{u}-I d\right)_{\mid F_{1} \oplus F_{2}}$. As a consequence, $T \notin \operatorname{Ker}\left(A_{u}-I d\right)_{\mid F_{1} \oplus F_{2}}^{\perp}$, hence there exists $u \in \operatorname{Ker}\left(A_{u}-I d\right)_{\mid F_{2}}$ such that $\langle T, u\rangle_{p, q} \neq 0$. But $\operatorname{Ker}\left(A_{u}-I d\right)_{\mid F_{2}}=\operatorname{Ker}\left(e^{\lambda} A-e^{2 \lambda} I d\right)$, so that rescaling $u$ if necessary, we get the desired proposition. $\diamond$

As a consequence of lemmas 4.4 and 4.3 , we get that if $h$ does not fix any point of $\mathbf{R}^{p, q}$, there exists $u \in \mathbf{R}^{p, q}$ such that for $s \geq 0, k \in \mathbf{N}$ :

$$
h^{k} \cdot \beta_{u}(s)=\beta_{u}\left(\frac{s}{1+k s}\right)
$$

If $\delta>0$ is small enough, there exists $\alpha:]-\delta, \delta\left[\rightarrow M\right.$, with $\alpha(0)=x_{0}$ and $\mathcal{D}_{x_{0}}^{b_{0}}(\alpha)(s)=\beta_{u}(s)$ for $\left.s \in\right]-\delta, \delta[$.

We then use the following reparametrizaton lemma:

Lemma 4.5. FM1, Proposition 5.3] Let I be an interval of $\mathbf{R}$ containing 0 , and $\alpha: I \rightarrow M a C^{1}$-curve such that $\alpha(0)=x_{0}$. We call $\beta:=\mathcal{D}_{x_{0}}^{b_{0}}(\alpha)$. We assume there exists for every $k \in \mathbf{N}$ a $C^{1}$-curve $f_{k}: I \rightarrow I$ satisfying $f_{k}(0)=0$, and such that $h^{k} \cdot \beta(s)=\beta\left(f_{k}(s)\right)$, for $s \in I$. Then, $\phi_{X}^{k} \cdot \alpha(s)=$ $\alpha\left(f_{k}(s)\right)$ for $s \in I$. 
Proof: we give an upshot of the proof for the reader's convenience. Let $\hat{\alpha}$ be a lift of $\alpha$ such that $\hat{\alpha}(0)=b_{0}$, and let $\hat{\beta}:=\mathcal{D}_{b_{0}}(\hat{\alpha})$. By hypothesis, there exists a $C^{1}$-curve $p_{k}: I \rightarrow P$, such that $\left(\operatorname{Ad} h^{k}\right) \cdot \hat{\beta}(s) \cdot p_{k}(s)=\hat{\beta}\left(f_{k}(s)\right)$ for every $s \in I$. On the other hand, $\mathcal{D}_{b_{0}}\left(\hat{\alpha} \circ f_{k}\right)=\hat{\beta} \circ f_{k}$ and $\mathcal{D}_{b_{0}}\left(\phi_{X}^{k} \cdot \hat{\alpha} \cdot h^{-k} \cdot p_{k}\right)=$ $\left(\operatorname{Ad} h^{k}\right) \cdot \hat{\beta} \cdot p_{k}=\hat{\beta} \circ f_{k}$. The two curves $\hat{\alpha} \circ f_{k}$ and $\phi_{X}^{k} \cdot \hat{\alpha} \cdot h^{-k} \cdot p_{k}$ satisfy the same ODE with the same initial condition: they are equal. $\diamond$

Using lemma 4.5, we get that for $s \in\left[0, \delta\left[\right.\right.$ and $k \in \mathbf{N}, \phi_{X}^{k} \cdot \alpha(s)$ is well defined, and moreover:

$$
\phi_{X}^{k} \cdot \alpha(s)=\alpha\left(\frac{s}{1+k s}\right)
$$

We are in the situation where $\lim _{k \rightarrow \infty} \phi_{X}^{k} \cdot \alpha(s)=x_{0}$ for every $s \in[0, \delta[$, and $D_{x_{0}} \phi_{X}^{k}\left(\alpha^{\prime}(0)\right)=\alpha^{\prime}(0)$. Moreover $\alpha^{\prime}(0) \neq 0$, because $\beta_{u}^{\prime}(0) \neq 0$. The following lemma shows that this forbids $\left\{\phi_{X}^{t}\right\}$ being linearizable in a neighborhood of $x_{0}$.

Lemma 4.6. Let $\left\{A^{t}\right\}$ be a linear flow on $\mathbf{R}^{n}$. Assume that $s \mapsto \alpha(s)$, $s \in]-\delta, \delta\left[\right.$ is a $C^{1}$-curve such that $A^{k} . \alpha^{\prime}(0)=\alpha^{\prime}(0)$ for every $k \in \mathbf{N}$, and $\lim _{k \rightarrow+\infty} A^{k} . \alpha(s)=0$ for every $s \in\left[0, \delta\left[\right.\right.$. Then $\alpha^{\prime}(0)=0$.

Proof: we see $\left\{A^{t}\right\}$ as a linear flow on $\mathbf{C}^{n}$, and call $e^{t \lambda_{1}}, \ldots, e^{t \lambda_{r}}$ the eigenvalues of $A^{t}$, with $\Re e\left(\lambda_{1}\right) \leq \ldots \leq \Re e\left(\lambda_{r}\right)$. Let $m$ be the greatest integer between 1 and $r$ such that $\Re e\left(\lambda_{j}\right)<0$ whenever $j \leq m$. For $i=$ $1, \ldots, r$, we call $C_{i}$ the characteristic subspace associated to $e^{t \lambda_{i}}$, namely $C_{i}:=\operatorname{Ker}\left(A^{t}-e^{t \lambda_{i}} I d\right)^{n}$. Those spaces do not depend on $t$, and $\mathbf{C}^{n}=$ $C_{1} \oplus \ldots \oplus C_{s}$. This yields a decomposition $\alpha(s)=\alpha_{1}(s)+\ldots \alpha_{r}(s)$, and the condition $\lim _{k \rightarrow \infty} A^{k} . \alpha(s)=0$ for $s \in\left[0, \delta\left[\right.\right.$ implies that $\alpha_{m+1}(s)=\ldots=$ $\alpha_{r}(s)=0$ for $s \in\left[0, \delta\left[\right.\right.$. In particular, $\alpha(s) \in C_{1} \oplus \ldots \oplus C_{m}$ for $s \in[0, \delta[$, and $\alpha^{\prime}(0) \in C_{1} \oplus \ldots \oplus C_{m}$ as well. We infer that $\lim _{k \rightarrow \infty} A^{k} . \alpha^{\prime}(0)=0$, and the relation $A^{k} \cdot \alpha^{\prime}(0)=\alpha^{\prime}(0)$ for all $k \in \mathbf{N}$ yields finally $\alpha^{\prime}(0)=0 . \diamond$

Remark 4.7. The previous proof shows that if $X$ is smooth, and $\left\{h^{t}\right\}$ does not fix any point in $\mathbf{R}^{p, q}$, then $X$ is not smoothly linearizable. actually, it shows a little bit more: the field $X$ is not even $C^{1}$-linearizable around $x_{0}$.

4.3. Holonomy and essentiality. We now characterize the local essentiality of $X$, thanks to the holonomy. Observe that in [Ca1, Theorem 2.1], 
M. S Capocci obtains the same kind of result, using also the normal Cartan bundle.

Proposition 4.8. The field $X$ is inessential in a neighborhood of $x_{0}$ if and only if its holonomy $\left\{h^{t}\right\} \subset\left(\mathbf{R}_{+}^{*} \times O(p, q)\right) \ltimes \mathbf{R}^{p, q}$ is conjugated in $P$ to a 1-parameter group of $O(p, q)$.

Proof: let us assume that $\left\{h^{t}\right\}$ is conjugated in $P$ to a 1-parameter group of $\mathrm{O}(p, q)$. Then, replacing $b_{0}$ by some $b_{0} . p$, we can assume that $\left\{h^{t}\right\} \subset \mathrm{O}(p, q)$. We choose $\mathcal{V}$ a neighborhood of 0 in $\mathfrak{n}^{-}$such that $\xi \mapsto \pi\left(\exp \left(b_{0}, \xi\right)\right)$ is a diffeomorphism from $\mathcal{V}$ on a neighborhood $U$ of $x_{0}$. Let us call $\Sigma:=$ $\exp \left(b_{0}, \mathcal{V}\right)$. We have a section $\sigma: U \rightarrow \Sigma$ defined by $\sigma\left(\pi\left(\exp \left(b_{0}, \xi\right)\right)\right):=$ $\exp \left(b_{0}, \xi\right), \forall \xi \in \mathcal{V}$. Observe that if $x=\exp \left(b_{0}, \xi\right)$ is a point of $U, \xi \in \mathcal{V}$, then for $t \in]-\delta, \delta[$ small enough, we have:

$$
\phi_{X}^{t} \cdot \sigma(x) \cdot h^{-t}=\phi_{X}^{t} \cdot \exp \left(b_{0}, \xi\right) \cdot h^{-t}=\exp \left(b_{0},\left(\operatorname{Ad} h^{t}\right) \cdot \xi\right)
$$

If we choosed $\delta$ small enough, $\exp \left(b_{0},\left(\operatorname{Ad} h^{t}\right) \cdot \xi\right)$ is a point of $\Sigma$ projecting on $\phi_{X}^{t} \cdot x$. Thus, it must be $\sigma\left(\phi_{X}^{t} \cdot x\right)$ and we get the equivariance relation:

$$
\phi_{X}^{t} \cdot \sigma(x) \cdot h^{-t}=\sigma\left(\phi_{X}^{t} \cdot x\right) .
$$

Now, let us fix $\lambda$ a type- $(p, q)$ scalar product on $\mathfrak{g} / \mathfrak{p}$, invariant for the adjoint action of $\mathrm{O}(p, q)$. We define on $U$ a type- $(p, q)$ metric $\mu$ by the following formula:

$$
\mu_{x}\left(\iota_{\sigma(x)}(\zeta), \iota_{\sigma(x)}(\zeta)\right):=\lambda(\zeta, \zeta)
$$

Here $\iota_{\sigma(x)}: \mathfrak{g} / \mathfrak{p} \rightarrow T_{x} M$ is the isomorphism introduced in section 2.4, By the construction of the normal Cartan bundle (see section 2.4), this metric $\mu$ is in the conformal class $[g]_{\mid U}$. We are going to show it is invariant by the local flow $\left\{\phi_{X}^{t}\right\}$, which will prove that $X$ is a Killing field for $\mu$. Let us recall the equivariance relation:

$$
\iota_{b . p}(\zeta)=\iota_{b}((\operatorname{Ad} p) \cdot \zeta)
$$

for $b \in B$, which implies:

$$
D_{x} \phi_{X}^{t}\left(\iota_{b}(\zeta)\right)=\iota_{\phi_{X}^{t} . b}(\zeta)
$$

for $b \in B$ in the fiber of $x$. We can compute, using (7), (8) and (9):

$$
\begin{gathered}
\mu_{\phi_{X}^{t} \cdot x}\left(D_{x} \phi_{X}^{t}\left(\iota_{\sigma(x)}(\zeta)\right), D_{x} \phi_{X}^{t}\left(\iota_{\sigma(x)}(\zeta)\right)\right)=\mu_{\phi_{X}^{t} \cdot x}\left(\iota_{\phi_{X}^{t} \cdot \sigma(x)}(\zeta), \iota_{\phi_{X}^{t} \cdot \sigma(x)}(\zeta)\right) \\
=\mu_{\phi_{X}^{t} \cdot x}\left(\iota_{\phi_{X}^{t} \cdot \sigma(x) \cdot h^{-t}}\left(\left(\operatorname{Ad} h^{t}\right) \cdot \zeta\right), \iota_{\phi_{X}^{t} \cdot \sigma(x) \cdot h^{-t}}\left(\left(\operatorname{Ad} h^{t}\right) \cdot \zeta\right)\right) \\
=\mu_{\phi_{X}^{t} \cdot x}\left(\iota_{\sigma\left(\phi_{X}^{t} \cdot x\right)}\left(\left(\operatorname{Ad} h^{t}\right) \cdot \zeta\right), \iota_{\sigma\left(\phi_{X}^{t} \cdot x\right)}\left(\left(\operatorname{Ad} h^{t}\right) \cdot \zeta\right)\right)
\end{gathered}
$$




$$
\left.=\lambda\left(\left(\operatorname{Ad} h^{t}\right) \cdot \zeta,\left(\operatorname{Ad} h^{t}\right) \cdot \zeta\right)\right)=\lambda(\zeta, \zeta)=\mu_{x}\left(\iota_{\sigma(x)}(\zeta), \iota_{\sigma(x)}(\zeta)\right)
$$

Reciprocally, if $X$ is inessential on a neighborhood $U$ of $x_{0}$, i.e $X$ is a Killing field for a metric $\mu \in[g]_{\mid U}$, then $X$ is linearizable at $x_{0}$. By the proposition 4.2, $\left\{h^{t}\right\}$ must fix a point of $\mathbf{R}^{p, q}$. This means there exists $p \in P$ such that $p . h^{t} . p^{-1} \in \mathbf{R}_{+}^{*} \times \mathrm{O}(p, q)$ for all $t \in \mathbf{R}$. Thus, replacing $b_{0}$ by $b_{0} . p$, we can assume that $\left\{h^{t}\right\} \subset \mathbf{R}_{+}^{*} \times \mathrm{O}(p, q)$. Now, we define on $\mathfrak{g} / \mathfrak{p}$ a type- $(p, q)$ scalar product $\nu$ by the formula:

$$
\nu(\zeta, \zeta):=\mu_{x_{0}}\left(\iota_{b_{0}}(\zeta), \iota_{b_{0}}(\zeta)\right), \zeta \in \mathfrak{g} / \mathfrak{p}
$$

Using again (7), (8) and (9), we get that for every $\zeta \in \mathfrak{g} / \mathfrak{p}$ :

$$
\begin{gathered}
\left.\nu\left(\left(\operatorname{Ad} h^{t}\right) \cdot \zeta,\left(\operatorname{Ad} h^{t}\right) \cdot \zeta\right)=\mu_{x_{0}}\left(\iota_{b_{0}}\left(\left(\operatorname{Ad} h^{t}\right) \cdot \zeta\right),\left(\operatorname{Ad} h^{t}\right) \cdot \zeta\right)\right) \\
=\mu_{x_{0}}\left(D_{x_{0}} \phi_{X}^{t}\left(\iota_{b_{0}}(\zeta)\right), D_{x_{0}} \phi_{X}^{t}\left(\iota_{b_{0}}(\zeta)\right)\right) \\
=\mu_{x_{0}}\left(\iota_{b_{0}}(\zeta), \iota_{b_{0}}(\zeta)\right)=\nu(\zeta, \zeta)
\end{gathered}
$$

We infer that $\nu$ is $\left(\operatorname{Ad} h^{t}\right)$-invariant, what implies that $\left(\operatorname{Ad} h^{t}\right)$ has determinant 1. The projection of $\left\{h^{t}\right\}$ on the $\mathbf{R}_{+}^{*}$-factor in $\mathbf{R}_{+}^{*} \times \mathrm{O}(p, q)$ must then be trivial, so that $\left\{h^{t}\right\} \subset \mathrm{O}(p, q)$. $\diamond$

\section{LoCAl CONFORMal Dynamics of Stable SEQUENCES}

In this section, $(M,[g])$ denotes a smooth, type- $(p, q)$ conformal pseudoRiemanniann structure of dimension $n \geq 3$. We call $(M, B, \omega)$ the corresponding normal Cartan bundle.

5.1. Holonomy of a sequence of conformal embeddings. The material below is essentially borrowed from [Fr4, section 4]. More details can be found in this reference.

Let $U$ be an open subset of $M$ and $f_{k}: U \rightarrow M$ a sequence of conformal embeddings. Let $x \in U$ be a point, and we assume that $f_{k}(x)$ is relatively compact in $M$. One says that a sequence $\left(h_{k}\right)$ of $P$ is a holonomy sequence of $\left(f_{k}\right)$ at $x$ if there exists a sequence $\left(b_{k}\right)$ in the fiber of $x$, contained in a compact set of $B$, and such that $f_{k}\left(b_{k}\right) \cdot h_{k}^{-1}$ is also contained in a compact set of $B$.

Let us begin with some remarks. First of all, there always exists a holonomy sequence associated to $\left(f_{k}\right)$. Also, the notion of holonomy sequence is stable by "compact perturbation": if $\left(h_{k}\right)$ is a holonomy sequence of $\left(f_{k}\right)$ at $x$, then so is any sequence $h_{k}^{\prime}=l_{1}(k) h_{k} l_{2}(k)$, where $l_{1}(k)$ and $l_{2}(k)$ are relatively 
compact sequences of $P$. One then says that $\left(h_{k}\right)$ and $\left(h_{k}^{\prime}\right)$ are equivalent. Since the action of $P$ on $B$ is proper, it is easily checked that reciprocally, any two holonomy sequences of $\left(f_{k}\right)$ at $x$ are always equivalent. Thus, what is really meaningfull is the equivalence class of holonomy sequences of $\left(f_{k}\right)$ at a point $x$. In what follows, when we will say "let $\left(h_{k}\right)$ be the holonomy of $\left(f_{k}\right)$ at $x$ ", we will mean that $\left(h_{k}\right)$ is a representative of the equivalence class of holonomy sequences at $x$. For convenience, we will often be led to change a holonomy sequence into another equivalent one.

5.2. Notion of stability. Let $U$ be an open subset of $M$ and $f_{k}: U \rightarrow M$ a sequence of conformal embeddings.

Definition 5.1 (Stability). One says that the sequence $\left(f_{k}\right)$ is stable at $x \in U$ if for every sequence $\left(x_{k}\right)$ of $U$ converging to $x, f_{k}\left(x_{k}\right)$ has a same limit $x_{\infty} \in M$. The sequence $\left(f_{k}\right)$ is said to be strongly stable at $x$ if there exists a neighborhood $V \subset U$ containing $x$ such that $f_{k}(\bar{V})$ converges to $x_{\infty} \in M$ for the Hausdorff topology.

We will also introduce a notion of stability for sequences in $P$ :

Definition 5.2. A sequence $\left(h_{k}\right)$ of $P$ is said to be stable if it is a sequence of $A^{+}$. Equivalently, $\left(h_{k}\right)$ is stable if it can be written:

$$
h_{k}=\operatorname{diag}\left(\lambda_{1}(k), \ldots, \lambda_{n}(k)\right) \in \mathbf{R}_{+}^{*} \times O(p, q),
$$

with $\lambda_{1}(k) \geq \ldots \geq \lambda_{n}(k) \geq 1$. The sequence $\left(h_{k}\right)$ is said to be strongly stable if moreover all the sequences $\frac{1}{\lambda_{i}(k)}$ tend to 0 .

The following lemma shows that the stability at $x$ of a sequence $\left(f_{k}\right)$ as above is encoded in its holonomy at $x$. In particular, a sequence $\left(h_{k}\right)$ of $P$, that we see as a sequence of conformal transformations of $\operatorname{Ein}^{p, q}$, is stable at $o$ (in the sense of definition 5.1) if and only if it is stable in the sense of definition 5.2. There is thus no ambiguity in the terminology.

Lemma 5.3. [Fr4, Lemma 4.3] The sequence $\left(f_{k}\right)$ is stable (resp. strongly stable) at $x \in U$ if and only if $f_{k}(x)$ converges to $x_{\infty} \in M$ and if there exists at $x$ a holonomy sequence $\left(h_{k}\right)$ which is stable (resp. strongly stable).

\section{Semi-Completeness properties for CONFormal VeCtor FieldS}

We are still considering a smooth, type- $(p, q)$ conformal structure $(M,[g])$, $p+q \geq 3$. We denote by $(M, B, \omega)$ the corresponding normal Cartan bundle. 
We assume that there exists on $M$ a conformal vector field $X$, having a singularity $x_{0} \in M$. We choose $b_{0} \in B$ above $x_{0}$ and call $\left\{h^{t}\right\}$ the holonomy flow of $X$ at $x_{0}$ relatively to $b_{0}$. We are going to use the work done so far to exhibit conditions on $\left\{h^{t}\right\}$ ensuring that some integral curves of $X$ in $M$ are semi-complete, i.e defined on $[0, \infty[$ or $]-\infty, 0]$.

\subsection{First semi-completeness properties.}

Proposition 6.1. There exists a real $R_{0}>0$ such that if $\alpha:[0,1] \rightarrow M$ is a conformal geodesic emanating from $x_{0}$, and if $\beta:=\mathcal{D}_{x_{0}}^{b_{0}}(\alpha)$ satisfies $h^{t} .[\beta] \subset B\left(o, R_{0}\right)$ for every $t \geq 0$, then $\phi_{X}^{t} . \alpha(u)$ is defined for every $t \geq 0$ and $u \in[0,1]$.

Proof: we first fix $W$ a relatively compact neighborhood of $x_{0}$ in $M$, and we denote by $r_{W}$ the real number given by lemma 3.1. Then we choose $R_{0}>0$ such that $8 n R_{0}<r_{W}$. Proposition 3.2 and lemma 3.1 ensure that if $[\gamma]$ is a conformal geodesic segment emanating from $x_{0}$, and if $\mathcal{D}_{x_{0}}^{b_{0}}([\gamma])=[\zeta]$ is included in $B\left(o, R_{0}\right)$, then $[\gamma] \subset W$.

Let us now take a geodesic segment $[\alpha]$ emanating from $x_{0}$, such that $[\beta]:=$ $\mathcal{D}_{x_{0}}^{b_{0}}([\alpha])$ satisfies $h^{t} .[\beta] \subset B\left(o, R_{0}\right)$ for every $t \geq 0$. In particular $[\alpha] \subset W$. We are going to show that if the flow $\left\{\phi_{X}^{t}\right\}$ is defined on some interval $[0, T[$, $T>0$ at each point of $\alpha$, then $\phi_{X}^{t} \cdot[\alpha] \subset W$ for every $t \in[0, T[$. Because $W$ is relatively compact, this will prove that $\phi_{X}^{t}$ is defined for every $t \geq 0$ at each point of $[\alpha]$. Since $[\beta]=\mathcal{D}_{x_{0}}^{b_{0}}([\alpha])$ and $\left\{h^{t}\right\}$ is the holonomy flow of $X$ at $b_{0}$, we have for every $t \in\left[0, T\left[, \mathcal{D}_{x_{0}}^{b_{0}}\left(\phi_{X}^{t} \cdot[\alpha]\right)=h^{t} \cdot[\beta]\right.\right.$. But by assumption $h^{t}$. $[\beta] \subset B\left(o, R_{0}\right)$ for $t \geq 0$, and in particular for $t \in[0, T$ [ so that we get $\phi_{X}^{t} \cdot[\alpha] \subset W$ for every $t \in[0, T[$, concluding the proof. $\diamond$

From this proposition, we infer:

Corollary 6.2. Let $\mathfrak{I}_{x_{0}}$ be the Lie algebra of conformal vector fields on $M$ vanishing at $x_{0}$, and let $\mathfrak{h} \subset \mathfrak{I}_{x_{0}}$ be a subalgebra. Let $H_{h} \subset P$ be the holonomy group of $\mathfrak{h}$ with respect to $b_{0}$. If $H_{h}$ is relatively compact in $P$, then there exists a neighborhood $U$ of $x_{0}$ on which the local action of $\mathfrak{h}$ integrates into the action of a connected Lie subgroup $H \subset$ Conf $(U)$, which is relatively compact in Conf $(U)$.

Proof: observe first that any compact subgroup of $P$ is conjugated in $P$ to a subgroup of $\mathrm{O}(p, q)$. Thus, replacing $b_{0}$ by a suitable $b_{0} . p$, we will assume 
that $\left(\operatorname{Ad} H_{h}\right)$ leaves $\mathfrak{n}^{-}$invariant. We define $\mathcal{U}_{r}:=\bigcup_{h \in H_{h}}(\operatorname{Ad} h) \cdot \mathcal{B}(0, r)$. This is an open subset of $\mathfrak{n}^{-}$and because $H_{h}$ is relatively compact, $\lim _{r \rightarrow 0} \mathcal{U}_{r}=$ $\{0\}$ (the limit being taken for the Hausdorff topology). Let $r>0$ be small enough so that on the one hand $\xi \mapsto \pi\left(\exp \left(b_{0}, \xi\right)\right)$ is a diffeomorphism from $\mathcal{U}_{r}$ onto its image $U$, and on the other hand $\pi_{G}\left(\exp \left(\mathcal{U}_{r}\right)\right) \subset B\left(o, R_{0}\right)$, where $R_{0}>0$ is given by proposition 6.1.

We first show that the local action of $\mathfrak{h}$ integrates into the action of a Lie group on $U$. This amounts to show that any vector field $X \in \mathfrak{h}$ is complete on $U$. Let $X \in \mathfrak{h}$ and $\left\{h^{t}\right\}$ its holonomy flow with respect to $b_{0}$. We have, for every $u \in[0,1]$, and every $\xi \in \mathcal{U}_{r}$ :

$$
\mathcal{D}_{x_{0}}^{b_{0}}(\alpha)(u)=\pi_{G}(\exp (u \xi)),
$$

where $\alpha(u):=\pi\left(\exp \left(b_{0}, u \xi\right)\right)$. Also, $h^{t} \cdot \pi_{G}(\exp (u \xi))=\pi_{G}\left(\exp \left(u\left(\operatorname{Ad} h^{t}\right)\right) \cdot \xi\right)$. Since $\left(\operatorname{Ad} h^{t}\right) \cdot \mathcal{U}_{r}=\mathcal{U}_{r}$, we obtain that $h^{t} \cdot \pi_{G}(\exp (u \xi)) \in B\left(o, R_{0}\right)$ for all $u \in[0,1], t \geq 0$. Proposition 6.1 then ensures that for every $x \in U, \phi_{X}^{t} \cdot x$ is defined for every $t \geq 0$. Considering $-X$ instead of $X$, we obtain in the same way that $\phi_{X}^{t} \cdot x$ is defined for every $t \leq 0$.

Let us call $H \subset \operatorname{Conf}(U)$ the connected Lie subgroup having $\mathfrak{h}_{\mid U}$ as Lie algebra. We are going to show that $H$ is relatively compact in Conf $(U)$, for the topology of uniform convergence on compact subsets of $U$. Let $\left(\phi_{k}\right)$ be a sequence of $H$. There exists an holonomy sequence $\left(h_{k}\right) \subset H$ such that $\phi_{k} \cdot b_{0} \cdot h_{k}^{-1}=b_{0}$. By assumption on $H$, there exists a subsequence $\left(h_{k_{j}}\right)$ which converges to $l \in P$.

The relation:

$$
\phi_{k_{j}} \cdot \exp \left(b_{0}, \xi\right) \cdot h_{k_{j}}=\exp \left(b_{0},\left(\operatorname{Ad} h_{k_{j}}\right) \cdot \xi\right)
$$

shows that $\left(\phi_{k_{j}}\right)$ converges uniformly on the compact subsets of $U$ to the diffeomorphism:

$$
\pi\left(\exp \left(b_{0}, \xi\right)\right) \mapsto \pi\left(\exp \left(b_{0} \cdot(\operatorname{Ad} l) \cdot \xi\right)\right), \xi \in \mathcal{U}_{r}
$$

(actually the convergence is $C^{\infty}$ ). We conclude that $H$ is relatively compact in the conformal group of $U$. $\diamond$

\subsection{Stability and semi-completeness for conformal vector fields.}

Proposition 6.1 shows how some properties of the holonomy give rise to semicomplete orbits for a conformal vector field. The good point with the notion of stability introduced in section 5 is that starting from a semi-completeness property for one orbit, it yields a semi-completeness property on a non empty 
open set of orbits. One can then study the dynamical behavior of $\left\{\phi_{X}^{t}\right\}$ on this open set, and try to infer some geometrical consequences. We keep in the statement below the notations introduced so far.

Proposition 6.3. Let $\alpha:[0,1] \rightarrow M$ be a conformal geodesic emanating from $x_{0}, x:=\alpha(1)$, and $\beta:=\mathcal{D}_{x_{0}}^{b_{0}}(\alpha)$. We assume that $\phi_{X}^{t} . \alpha(u)$ is defined for every $(t, u) \in \mathbf{R}_{+} \times[0,1]$, that $\lim _{t \rightarrow \infty} h^{t} .[\beta]=o$, and that for every sequence $t_{k} \rightarrow \infty,\left(h^{t_{k}}\right)$ is stable (resp. strongly stable) at $\beta(1)$. Then

(1) There exists an open set $V$ containing $x$ such that for every $y \in V$, $\phi_{X}^{t} . y$ is defined for every $t \geq 0$.

(2) Moreover there exists a sequence $\left(s_{k}\right)$ of $\mathbf{R}_{+}$tending to infinity, such that for every $y \in V, \lim _{k \rightarrow \infty} \phi_{X}^{s_{k}} . y$ exists, $\left(\phi_{X}^{s_{k}}\right)$ is stable at $y$ (resp. $\phi_{X}^{s_{k}} . V \rightarrow x_{0}$, and $\left(\phi_{X}^{s_{k}}\right)$ is strongly stable at each point of $\left.V\right)$, and the holonomy of $\left(\phi_{X}^{s_{k}}\right)$ at $y$ is that of $\left(h^{s_{k}}\right)$ at $\beta(1)$.

Proof: by corollary 3.3, we have $\lim _{t \rightarrow \infty} \phi_{X}^{t} \cdot \alpha(u)=x_{0}$ for every $u \in[0,1]$. Let us fix a compact set $K$ of $M$ containing $\left\{\phi_{X}^{t} \cdot x\right\}_{t \geq 0} \cup\left\{x_{0}\right\}$ in its interior. We want to show that there exists an open neighborhood $V$ of $x$ having the property that for every $t_{0}>0$ such that $\phi_{X}^{t}$ is defined on $\left[0, t_{0}\right.$ [ at each point of $V$, we have actually $\phi_{X}^{t}(V) \subset K, \forall t \in\left[0, t_{0}[\right.$.

If it is not the case, we can find a sequence $\left(y_{k}\right)$ tending to $x$, as well as times $t_{k} \in\left[0, \infty\left[\right.\right.$ such that $\phi_{X}^{t} . y_{k}$ is defined on $\left[0, t_{k}\right]$, but $\phi_{X}^{t_{k}} \cdot y_{k} \notin K$. We are now going to use the following lemma, the proof of which we postpone a little bit later:

Lemma 6.4. Let us assume there exists a conformal geodesic $\alpha:[0,1] \rightarrow M$ emanating from $x_{0}$ such that for every $u \in[0,1], \phi_{X}^{t} . \alpha(u)$ is defined for every $t \geq 0($ resp. $t \leq 0)$, and $\lim _{t \rightarrow \infty} h^{t} .[\beta]=o\left(\right.$ resp. $\left.\lim _{t \rightarrow-\infty} h^{t} .[\beta]=o\right)$, where $\beta:=\mathcal{D}_{x_{0}}^{b_{0}}(\alpha)$. Then for every sequence $t_{k} \rightarrow \infty$ (resp. $\left.t_{k} \rightarrow-\infty\right)$, and every $u \in[0,1]$, the holonomy of $\left(\phi_{X}^{t_{k}}\right)$ at $\alpha(u)$ is the holonomy of $\left(h^{t_{k}}\right)$ at $\beta(u)$.

This lemma tells us that the holonomy $\left(h_{k}\right)$ of $\left(h^{t_{k}}\right)$ at $\beta(1)$ is also an holonomy sequence of $\left(\phi_{X}^{t_{k}}\right)$ at $x$. Because $\left(h^{t_{k}}\right)$ is stable at $\beta(1)$, we can assume that $\left(h_{k}\right)$ is a sequence of $A^{+}$that we write $h_{k}=\operatorname{diag}\left(\lambda_{1}(k), \ldots, \lambda_{n}(k)\right)$. Let $\left(b_{k}\right)$ be a relatively compact sequence of $B$, such that $\phi_{X}^{t_{k}}\left(b_{k}\right) \cdot h_{k}^{-1}$ is also relatively compact in $B$. Because $\left(y_{k}\right)$ tends to $x$, there is a sequence $\left(\xi_{k}\right)$ of $\mathfrak{n}^{-}$tending to 0 , and satisfying $\pi\left(\exp \left(b_{k}, \xi_{k}\right)\right)=y_{k}$. We have:

$$
\phi_{X}^{t_{k}}\left(\exp \left(b_{k}, \xi_{k}\right) \cdot h_{k}^{-1}\right)=\exp \left(\phi_{X}^{t_{k}}\left(b_{k}\right) \cdot h_{k}^{-1},\left(\operatorname{Ad} h_{k}\right) \cdot \xi_{k}\right)
$$


The stability of $\left(h^{t_{k}}\right)$ at $\beta(1)$ means that $\left|\frac{1}{\lambda_{i}(k)}\right|, i=1, \ldots, n$ are bounded sequences, ensuring that $\left(\operatorname{Ad} h_{k}\right) \cdot \xi_{k}$ tends to 0 . Projecting on $M$, we get that $\phi_{X}^{t_{k}}\left(y_{k}\right)$ tends to $x_{0}$. But $x_{0}$ is in the interior of $K$, contradicting $\phi_{X}^{t_{k}}\left(y_{k}\right) \notin K$. This shows the first point of the proposition.

Let us now prove the second point. We first choose a sequence $\left(t_{k}\right)$ tending to infinity. By the first point, we know that $\phi_{X}^{t_{k}}$ is well defined on a neighborhood of $x$ for all $k \in \mathbf{N}$, and lemma 6.4 ensures that $\left(\phi_{X}^{t_{k}}\right)$ is stable (resp. strongly stable) at $x$. In particular, it is equicontinuous and we can apply the theorem 1.1 of [Fr4]: replacing if necessary $V$ by one of its open subsets, there exists a sequence $\left(s_{k}\right)$ tending to infinity, which is a subsequence of $\left(t_{k}\right)$, and such that $\left(\phi_{X}^{s_{k}}\right)$ tends uniformly on the compact subsets of $V$ to a smooth map $\phi: V \rightarrow M$. In particular, for every $y \in M, \lim _{k \rightarrow \infty} \phi_{X}^{s_{k}} . y$ exists. Lemma 6.1 of [Fr4] ensures that the holonomy $\left(h_{k}\right)$ of $\left(\phi_{X}^{s_{k}}\right)$ at $x$ is an holonomy sequence of $\left(\phi_{X}^{s_{k}}\right)$ at $y$ for every $y \in V$. Moreover, lemma 6.4 says that this holonomy $\left(h_{k}\right)$ is that of $\left(h^{s_{k}}\right)$ at $\beta(1)$. $\diamond$

6.2.1. Proof of Lemma 6.4. We write $\alpha(u)=\pi\left(\exp \left(b_{0}, u \xi\right)\right)$. By hypothesis, $u \mapsto \beta_{k}(u):=h^{t_{k}} \cdot \pi_{G}(\exp (u \xi))$ is a sequence of conformal geodesics tending to $o$ for the Hausdorff topology. Proposition 3.2 ensures that the length $L^{o}\left(\beta_{k}\right)$ of those curves tends to 0 as $k \rightarrow \infty$. For each $k \in \mathbf{N}$, there is a $C^{1}$-curve $p_{k}:[0,1] \rightarrow P$, with $p_{k}(0)=1_{G}$, such that $\hat{\beta}_{k}(u):=$ $\exp \left(u\left(\operatorname{Ad} h^{t_{k}}\right) \cdot \xi\right) \cdot p_{k}(u)^{-1}$ is a curve of $N^{-}$. As already noticed, the length of $\hat{\beta}_{k}$ with respect to $\rho^{G}$ is just $L^{o}\left(\beta_{k}\right)$. We thus get

$$
\lim _{k \rightarrow \infty} h^{t_{k}} \cdot \exp (u \xi) \cdot\left(p_{k}(u) h^{t_{k}}\right)^{-1}=1_{G}
$$

proving that $\left(p_{k}(u) h^{t_{k}}\right)$ is a holonomy sequence of $\left(h^{t_{k}}\right)$ at $\beta(u)=\pi_{G}(\exp (u \xi))$. On the other hand, if we define $\hat{\alpha}_{k}(u):=\phi_{X}^{t_{k}} \cdot \exp \left(b_{0}, u \xi\right) \cdot\left(p_{k}(u) h^{t_{k}}\right)^{-1}$, we also have $\hat{\beta}_{k}=\mathcal{D}_{x_{0}}^{b_{0}}\left(\hat{\alpha}_{k}\right)$. Thus the length of $\hat{\alpha}_{k}$ relatively to $\rho^{B}$ tends to 0 because it is the length of $\hat{\beta}_{k}$ relatively to $\rho^{G}$. Hence

$$
\lim _{k \rightarrow \infty} \phi_{X}^{t_{k}} \cdot \exp \left(b_{0}, u \xi\right) \cdot\left(p_{k}(u) h^{t_{k}}\right)^{-1}=b_{0}, \text { for every } u \in[0,1]
$$

This shows that $\left(p_{k}(u) h^{t_{k}}\right)$ is also a holonomy sequence for $\left(\phi_{X}^{t_{k}}\right)$ at $x_{0}$.

\section{Application to Riemannian conformal VeCtor fields: PROOF OF THEOREM 1.2}

Here, $(M,[g])$ is a smooth Riemannian conformal structure of dimension $\geq 3$. We still denote by $(M, B, \omega)$ the corresponding normal Cartan bundle. Let 
us point out that in the Riemannian framework, the space $\operatorname{Ein}^{0, n}$ is nothing but the standard conformal sphere $\mathbf{S}^{n}$, and $\mathbf{R}^{0, n}$ is the Euclidean space $\mathbf{R}^{n}$. We consider $x_{0} \in M$, and $\mathfrak{I}_{x_{0}}$ the Lie algebra of smooth conformal vector fields on $M$ vanishing at $x_{0}$. We will call $\mathfrak{I}_{h}$ the associated holonomy algebra with respect to some point $b_{0} \in B$ in the fiber of $x_{0}$, and $I_{h} \subset P$ will denote the connected Lie subgroup associated to $\mathfrak{I}_{h}$.

The aim of this section is to prove the theorem below, of which theorem 1.2 is a particular case:

Theorem 7.1. Let $(M, g)$ be a smooth Riemannian manifold of dimension $n \geq 3$, and $x_{0} \in M$. Let $\mathfrak{I}_{x_{0}}$ be the Lie algebra of conformal vector fields vanishing at $x_{0}$, and $\mathfrak{h} \subset \mathfrak{I}_{x_{0}}$ a subalgebra. We denote by $\mathfrak{h}_{h}$ the holonomy algebra of $\mathfrak{h}$. Then :

(1) Either there is a neighborhood $U$ of $x_{0}$ on which the local action of $\mathfrak{h}$ integrates into the action of a relatively compact subgroup $H \subset$ Conf $(U)$. In this case $H$ preserves a metric in the conformal class $[g]_{\mid U}$, and its action around $x_{0}$ is linearizable.

(2) If we are not in the previous case, there is a neighborhood $U$ of $x_{0}$ which is conformally flat, and the Lie algebra $\mathfrak{h}$ is essential on each neighborhood of $x_{0}$.

In any case, there is a smooth diffeomorphism from a neighborhood of $x_{0}$ in $M$ onto a neighborhood of o in $\mathbf{S}^{n}$, conjugating $\mathfrak{h}$ and its holonomy algebra $\mathfrak{h}_{h}$.

Before going further, let us precise that a subalgebra $\mathfrak{h} \subset \mathfrak{I}_{x_{0}}$ is said to be inessential on some neighborhood $U$ of $x_{0}$ if there exists a metric $g$ in the conformal class $[g]_{\mid U}$ such that every vector field of $\mathfrak{h}$ is a Killing field for $g$ (i.e generates a local flow of isometries of $g$ ). If we are not in this case, the Lie algebra $\mathfrak{h}$ is said essential.

7.1. Strengthening of some results in the Riemannian setting. We begin by proving some technical results allowing to sharpen some of our previous statements in the Riemannian case.

Lemma 7.2. Let $m \geq 1$, and $(\mathbf{R} \oplus \mathfrak{o}(m)) \ltimes \mathbf{R}^{m}$ be the Lie algebra of $\left(\mathbf{R}_{+}^{*} \times O(m)\right) \ltimes \mathbf{R}^{m}$, the group of affine conformal transformations of $\mathbf{R}^{m}$. Let $\mathfrak{h}$ be a subalgebra of $(\mathbf{R} \oplus \mathfrak{o}(m)) \ltimes \mathbf{R}^{m}$, and $H$ the connected Lie subgroup of $\left(\mathbf{R}_{+}^{*} \times O(m)\right) \ltimes \mathbf{R}^{m}$ having $\mathfrak{h}$ as Lie algebra. 
(1) Assume that for every $Y \in \mathfrak{h}$, the 1-parameter subgroup $\{\exp (t Y)\}_{t \in \mathbf{R}}$ is relatively compact in $\left(\mathbf{R}_{+}^{*} \times O(m)\right) \ltimes \mathbf{R}^{m}$. Then $H$ is relatively compact in $\left(\mathbf{R}_{+}^{*} \times O(m)\right) \ltimes \mathbf{R}^{m}$.

(2) Assume that every element of $H$ has a fixed point in $\mathbf{R}^{m}$. Then $H$ has a fixed point in $\mathbf{R}^{m}$.

Proof: we begin with the first point, that we prove by induction. When $m=1$, the claim is obvious. Assume now $m>1$, and take $Y \in \mathfrak{h}$. Because the adjoint representation of $\{\exp (t Y)\}$ on $(\mathbf{R} \oplus \mathfrak{o}(m)) \ltimes \mathbf{R}^{m}$ is relatively compact, the eigenvalues of $(\operatorname{ad} Y)$ are purely imaginary, and the same is true for the eigenvalues of $(\operatorname{ad} Y)_{\mid \mathfrak{h}}$. As a consequence, if the center of $\mathfrak{h}$ is trivial, then the Killing form of $\mathfrak{h}$ is negative definite. It is then a classical result that $H$ is compact, as a Lie group, hence is a compact subgroup of $\left(\mathbf{R}_{+}^{*} \times \mathrm{O}(m)\right) \ltimes \mathbf{R}^{m}$.

If the center $\mathfrak{z} \subset \mathfrak{h}$ is nontrivial, and $Z \subset\left(\mathbf{R}_{+}^{*} \times \mathrm{O}(m)\right) \ltimes \mathbf{R}^{m}$ is the associated connected subgroup, then $\bar{Z}$, the closure of $Z$ into $\left(\mathbf{R}_{+}^{*} \times \mathrm{O}(m)\right) \ltimes \mathbf{R}^{m}$, is a compact torus. In particular, it must fix at least one point of $\mathbf{R}^{m}$, and the affine subspace $\mathcal{F} \subsetneq \mathbf{R}^{m}$ of fixed points of $\bar{Z}$ is acted upon conformally by $H$. Let $m^{\prime}$ be the dimension of $\mathcal{F}$. If $m^{\prime}=0$, then $H$ fixes a point in $\mathbf{R}^{m}$, hence is conjugated to a subgroup of $\mathbf{R}_{+}^{*} \times \mathrm{O}(m)$. Because all the 1parameter subgroups of $H$ are relatively compact, $H$ is actually conjugated to a subgroup of $\mathrm{O}(m)$, hence is relatively compact in $\left(\mathbf{R}_{+}^{*} \times \mathrm{O}(m)\right) \ltimes \mathbf{R}^{m}$. Now, if $m^{\prime} \geq 1$, we can use the induction hypothesis: the restriction $H_{\mid \mathcal{F}}$ is relatively compact into $\left(\mathbf{R}_{+}^{*} \times \mathrm{O}\left(m^{\prime}\right)\right) \ltimes \mathbf{R}^{m^{\prime}}$. We infer that $H$ fixes a point of $\mathcal{F}$, and the same argument as above implies that $H$ is conjugated to a subgroup of $\mathrm{O}(m)$.

We now prove the second point of the lemma, arguing again by induction. For $m=1$, the claim is pretty clear. Assume now that $m>1$. We consider the Lie algebra homomorphism $\pi_{1}: \mathfrak{h}_{h} \subset(\mathbf{R} \oplus \mathfrak{o}(m)) \ltimes \mathbf{R}^{m} \rightarrow \mathbf{R}$, and call $\mathfrak{h}_{h}^{\prime}$ the kernel of $\pi_{1}$. If this kernel is trivial, then $H_{h}$ is either trivial, or is conjugated to a 1-parameter subgroup of $\mathbf{R}_{+}^{*} \times \mathrm{O}(m)$. In any case, it fixes a point of $\mathbf{R}^{m}$.

We assume now that $\mathfrak{h}_{h}^{\prime}$ is nontrivial, and call $H_{h}^{\prime}$ the connected subgroup of $\mathrm{O}(m) \ltimes \mathbf{R}^{m}$ with Lie algebra $\mathfrak{h}_{h}^{\prime}$. By hypothesis, if $Y \in \mathfrak{h}_{h}^{\prime}$, then $\{\exp (t Y)\}$ has a fixed point in $\mathbf{R}^{m}$. But a 1-parameter subgroup of $\mathrm{O}(m) \ltimes \mathbf{R}^{m}$ having a fixed point is conjugated to a 1-parameter subgroup of $\mathrm{O}(\mathrm{m})$, hence is 
relatively compact. We infer from the first point of the lemma that $H_{h}^{\prime}$ is relatively compact in $\mathrm{O}(m) \ltimes \mathbf{R}^{m}$, hence fixes a point in $\mathbf{R}^{m}$. We call $\mathcal{F} \subsetneq \mathbf{R}^{m}$ the affine subspace, of dimension $m^{\prime}<m$, comprising the fixed points of $H_{h}^{\prime}$. Because $H_{h}^{\prime}$ is normal in $H_{h}, \mathcal{F}$ is left invariant by $H_{h}$, and $\left(H_{h}\right)_{\mid \mathcal{F}}$ is a subgroup of $\left(\mathbf{R}_{+}^{*} \times \mathrm{O}\left(m^{\prime}\right)\right) \ltimes \mathbf{R}^{m^{\prime}}$. We are going to show that every 1-parameter subgroup $\{\exp (t Y)\}, Y \in \mathfrak{h}_{h}$, has a fixed point in $\mathcal{F}$. It is clear if $Y \in \mathfrak{h}_{h}^{\prime}$. Now, if $Y \in \mathfrak{h}_{h} \backslash \mathfrak{h}_{h}^{\prime}$, then $\{\exp (t Y)\}$ is conjugated to a subgroup $e^{\lambda t} A^{t}$, where $\lambda \neq 0$, and $\left\{A^{t}\right\} \subset \mathrm{O}(m)$. In particular, $\{\exp (t Y)\}$ has a unique fixed point $y_{0}$ in $\mathbf{R}^{n}$, having the property that $\lim _{t \rightarrow \infty} \exp (t Y) \cdot y=y_{0}$ for every $y \in \mathbf{R}^{m}$, or $\lim _{t \rightarrow-\infty} \exp (t Y) \cdot y=y_{0}$ for every $y \in \mathbf{R}^{m}$. Choosing $y \in \mathcal{F}$, and because $\mathcal{F}$ is a closed subset, we get $y_{0} \in \mathcal{F}$. We can then use the induction hypothesis and obtain that $H_{h}$ fixes a point in $\mathcal{F}$. This concludes the proof of the second point. $\diamond$

As a corollary of this lemma, we can strengthen the statements of propositions 4.2 and 4.8 .

Corollary 7.3. Let $\mathfrak{h} \subset \mathfrak{I}_{x_{0}}$ be a subalgebra of smooth conformal vector fields. Let $b_{0} \in B$ in the fiber of $x_{0}$ and $\mathfrak{h}_{h}\left(\right.$ resp. $\left.H_{h}\right)$ the holonomy algebra (resp. the holonomy group) of $\mathfrak{h}$ with respect to $b_{0}$. Then:

(1) The algebra $\mathfrak{h}$ is linearizable in a neighborhood of $x_{0}$ if and only if $H_{h}$ has a fixed point in $\mathbf{R}^{n}$. In this case, there is a smooth diffeomorphism from a neighborhood of $x_{0}$ in $M$ onto a neighborhood of o in $\mathbf{S}^{n}$ which conjugates $\mathfrak{h}$ and $\mathfrak{h}_{h}$.

(2) The algebra $\mathfrak{h}$ is inessential in a neighborhood of $x_{0}$ if and only if $H_{h} \subset P$ is conjugated into $P$ to a subgroup of $O(n)$.

Proof: we keep the notations of proposition 4.2. If $H_{h}$ fixes a point of $\mathbf{R}^{n}$, we may assume that this point is 0 . Then the smooth diffeomorphism $\psi \circ \varphi^{-1}$, from $U$ onto $V$ conjugates $\mathfrak{h}$ and $\mathfrak{h}_{h}$.

Reciprocally, if $\mathfrak{h}$ is smoothly linearizable around $x_{0}$, proposition 4.2 ensures that for every $Y \in \mathfrak{h}_{h}$, the 1-parameter group $\{\exp (t Y)\}_{t \in \mathbf{R}}$ has a fixed point in $\mathbf{R}^{n}$. Then we can apply the second point of lemma 7.2 and infer that $H_{h}$ fixes a point in $\mathbf{R}^{n}$.

We now prove the second point of the corollary. We can reproduce verbatim the proof of proposition 4.8 to get that whenever $H_{h}$ is conjugated to a subgroup of $\mathrm{O}(n)$, then $\mathfrak{h}$ is inessential in a neighborhood $U$ of $x_{0}$. Reciprocally, if $\mathfrak{h}$ is inessential in a neighborhood of $x_{0}$, it is smoothly linearizable around 
$x_{0}$. By the first point of the corollary, $H_{h}$ must fix a point of $\mathbf{R}^{n}$, hence is conjugated to a subgroup of $\mathbf{R}_{+}^{*} \times \mathrm{O}(n)$. Because each $X \in \mathfrak{h}$ is inessential around $x_{0}$, every 1-parameter group of $H_{h}$ has determinant 1 , showing that $H_{h}$ is actually conjugated to a subgroup of $\mathrm{O}(n)$.

We can now prove theorem 7.1 in the case when $H_{h}$ is relatively compact in $P$. In this case, $H_{h}$ has a fixed point in $\mathbf{R}^{n}$, so that by corollary 7.3 , $\mathfrak{h}$ is smoothly linearizable and there exists a smooth diffeomorphism $\varphi$ from a neighborhood $U$ of $x_{0}$ onto a neigborhood $V$ of $o$, conjugating $\mathfrak{h}$ and the holonomy algebra $\mathfrak{h}_{h}$. The second point of corollary 7.3 says that $\mathfrak{h}$ is inessential in a neighborhood of $x_{0}$. Finally, corollary 6.2 ensures that there exists a neighborhood $U$ of $x_{0}$ on which every vector field of $\mathfrak{h}$ is complete. In other words, the local action of $\mathfrak{h}$ integrates into the action of a Lie subgroup $H \subset$ Conf $(U)$. We are then in case (1) of theorem 7.1

Whenever $H_{h}$ is not relatively compact in $P$, lemma 7.2 ensures that for some $X_{h} \in \mathfrak{h}_{h}$, the 1-parameter group $\left\{h^{t}\right\}:=\left\{\exp \left(t X_{h}\right)\right\}$ is not relatively compact in $P$. There exists $X \in \mathfrak{h}$ such that $\left\{h^{t}\right\}$ is the holonomy flow of $X$ with respect to $b_{0}$. Up to conjugacy in $P$, the flow $\left\{h^{t}\right\}$ is of two possible kinds, and we are going to prove theorem 7.1 in each case.

7.2. The flow $\left\{h^{t}\right\}$ is a non relatively compact flow of $\mathbf{R}_{+}^{*} \times \mathbf{O}(n)$. The adjoint action of $h^{t}$ on $\mathfrak{n}^{-}$is by dilations for the metric $<,>_{\mathfrak{n}^{-}}$. In particular there exists $\lambda \neq 0$ such that $\left(\operatorname{Ad} h^{t}\right) \cdot \mathcal{B}(0, r)=\mathcal{B}\left(0, e^{\lambda t} r\right)$, for every $r>0$. Replacing if necessary $X$ by $-X$, we will assume that $\lambda<0$. Proposition 4.2 ensures that $X$ is locally conjugated in a neighborhood of $x_{0}$ to its holonomy field $X_{h}$, which is a field of linear contractions in $\mathbf{R}^{n}$. Thus, there exists a neighborhood $U$ of $x_{0}$ such that for every point $x \in U, \phi_{X}^{t} \cdot x$ is defined for every $t \geq 0$, and moreover $\lim _{t \rightarrow \infty} \phi_{X}^{t} \cdot U=x_{0}$. In particular the sequence $\left\{\phi_{X}^{k}\right\}_{k>0}$ is strongly stable at each point of $U$.

We conclude that $U$ is conformally flat thanks to the:

Lemma 7.4. Let $(M,[g])$ be a conformal Riemannian structure of dimension $n \geq 3$, and $f_{k}: U \rightarrow M$ a sequence of conformal embedings, which is strongly stable at $x \in U$. Then the Weyl tensor (resp. the Cotton tensor if $\operatorname{dim} M=3)$ vanishes on a neighborhood of $x$.

Proof: If $f_{k}: U \rightarrow M$ is strongly stable at $x$, then it is equicontinuous at $x$. We can then use [Fr2, Proposition 5] (the proof of which deals with conformal 
diffeomorphisms of $M$, but can be adapted to conformal embeddings in a straigthforward way) and get that the Weyl tensor (resp. the Cotton tensor if $\operatorname{dim} M=3$ ) vanishes at $x$. By definition, being strongly stable is an open property, so that the lemma follows. $\diamond$

We are thus in case (2) of theorem [7.1. Proposition 4.8 ensures that $X$, hence $\mathfrak{h}$, is essential on any neighborhood of $x_{0}$. By the remark 4.1, $\mathfrak{h}$ is smoothly conjugated (actually the conjugacy map is a smooth conformal diffeomorphism) to its holonomy algebra $\mathfrak{h}_{h}$.

\subsection{The flow $\left\{h^{t}\right\}$ is the commutative product of a translation flow} $\tau^{t}$, and a relatively compact flow $\kappa^{t}$ in $\mathbf{O}(n)$. For every $R>0$, and $z=\pi_{G}(\exp (\xi)) \in B(o, R), \xi \in \mathcal{B}(0, R)$, we call $[o z]$ the geodesic segment defined by:

$$
[o z]:=\left\{\pi_{G}(\exp (u \xi)) \mid u \in[0,1]\right\}
$$

Lemma 7.5. For every $R>0$ and $z \in B(o, R)$, we are in one of the following cases:

- For every $t \geq 0, h^{t} \cdot[o z] \subset B(o, R)$ and $\lim _{t \rightarrow \infty} h^{t} \cdot[o z]=o$.

- For every $t \leq 0, h^{t} .[o z] \subset B(o, R)$ and $\lim _{t \rightarrow-\infty} h^{t} .[o z]=o$.

Proof: with the notations of section 3.4, and in the Riemannian case, we have $\langle x, x\rangle=\|x\|^{2}=Q^{0, n}(x)$. Everywhere it is defined, the map $s(x)=\frac{-2 x}{<x, x>}$ satisfies:

$$
j^{o}(s(x))=j(x)
$$

We first prove the proposition when $h^{t}$ is the translation of vector $t v$, for a nonzero $v \in \mathbf{R}^{n}$. We write $z=j(x)$, and $z \in B(o, R)$ means that we have $\|s(x)\|<R$, i.e $\|x\|>\frac{2}{R}$. As a set, the segment $[o z]$ is:

$$
[o z]=\{o\} \cup\{j(u x) \mid u \in[1, \infty[\}
$$

From $\|s(u x+t v)\|^{2}=4\left(u^{2}\|x\|^{2}+t^{2}\|v\|^{2}+2 u t<x, v>\right)^{-1}$, we infer:

- if $\langle x, v\rangle \geq 0$, then $\|s(u x+t v)\|^{2} \leq \frac{4}{\|x\|^{2}}$ for every $t \geq 0$, meaning that $h^{t} .[o z] \subset B(o, R)$ for $t \geq 0$. Also, from $\sup _{u \in[1, \infty[}\|s(u x+t v)\|^{2} \leq \frac{1}{t^{2}\|v\|^{2}}$ for $t>0$, we get $\lim _{t \rightarrow \infty} \tau^{t}$. [oz] $=o$.

- if $\langle x, v\rangle \leq 0$, then $\|s(u x+t v)\|^{2} \leq \frac{4}{\|x\|^{2}}$ for every $t \leq 0$, meaning that $h^{t} \cdot[o z] \subset B(o, R)$ for $t \leq 0$. Also, from $\sup _{u \in[1, \infty[}\|s(u x+t v)\|^{2} \leq \frac{1}{t^{2}\|v\|^{2}}$ for $t<0$, we get $\lim _{t \rightarrow-\infty} \tau^{t} \cdot[o z]=o$. 
If now $\left\{h^{t}\right\}$ is the commutative product of $\left\{\tau^{t}\right\}$, the translation flow of direction $t v$, and of a flow $\left\{\kappa^{t}\right\}$ in $\mathrm{O}(n)$, then the lemma remains true since $\kappa^{t} \cdot[o z]=\left[o \kappa^{t} . z\right]$, and the balls $B(o, R)$ are left invariant under the action of $\kappa^{t} . \diamond$

Lemma 7.6. Let $\left(t_{k}\right)$ be a sequence of real numbers tending to $\infty$ (resp. to $-\infty)$. Then the sequence $\left(h^{t_{k}}\right)$ is strongly stable at each point $z \in j\left(\mathbf{R}^{n}\right)$.

Proof: we write that $h^{t_{k}}$ is a commutative product $\tau^{t_{k}} \cdot \kappa^{t_{k}}$, with $\tau^{t_{k}}$ the translation of vector $t_{k} v \neq 0$, and $\kappa^{t_{k}} \in \mathrm{O}(n)$. Let $z=j(x) \in j\left(\mathbf{R}^{n}\right)$ and $y$ be in the euclidean ball of center $x$ and radius $r>0$. Then:

$$
\lim _{k \rightarrow \infty}\left\|s\left(y+t_{k} v\right)\right\|^{2}=0
$$

uniformly on $B(x, r)$. As a consequence $\lim _{k \rightarrow \infty} \tau^{t_{k}} \cdot j(B(x, r))=o$. Because $\left\{\kappa^{t_{k}}\right\}$ has compact closure in $P$, we have also:

$$
\lim _{k \rightarrow \infty} h^{t_{k}} \cdot j(B(x, r))=o,
$$

showing that $\left(h^{t_{k}}\right)$ is strongly stable at $z . \diamond$

Let us consider $R_{0}$ the real number given by proposition 6.1, and let us choose $0<R<R_{0}$ small enough so that $\xi \mapsto \pi\left(\exp \left(b_{0}, \xi\right)\right)$ is defined and injective on $\mathcal{B}(0, R)$. Let us define $U:=\pi\left(\exp \left(b_{0}, \mathcal{B}(0, R)\right)\right)$. Pick $x \in U$, and write $x=\pi\left(\exp \left(b_{0}, \xi\right)\right)$ for $\xi \in \mathcal{B}(0, R)$. Setting $\alpha(u):=\pi\left(\exp \left(b_{0}, u \xi\right)\right)$, we see thanks to lemma 7.5 that $\beta:=\mathcal{D}_{x_{0}}^{b_{0}}(\alpha)$ satisfies either $h^{t} \cdot[\beta] \subset B\left(o, R_{0}\right)$ for every $t \geq 0$, and $\lim _{t \rightarrow \infty} h^{t} \cdot[\beta]=o$, or $h^{t}$. $[\beta] \subset B\left(o, R_{0}\right)$ for every $t \leq 0$, and $\lim _{t \rightarrow-\infty} h^{t} .[\beta]=o$. We will assume in the following that we are in the first of those two cases (the proof is the same for the second case). Proposition 6.1 ensures that $\phi_{X}^{t} \cdot \alpha(u)$ is defined for every $t \geq 0$ and $u \in[0,1]$. Corollary 3.3 yields $\lim _{t \rightarrow \infty} \phi_{X}^{t} \cdot \alpha(u)=x_{0}$ for every $u \in[0,1]$. We can now use proposition 6.3. there exists $V$ a neighborhood of $x$ in $U$ such that $\phi_{X}^{t}$ is defined on $V$ for every $t \geq 0$. On the other hand, the second point of proposition 6.3. together with the third point of lemma 7.6 ensures that for some sequence $s_{k} \rightarrow \infty,\left(\phi_{X}^{s_{k}}\right)$ is strongly stable at $x$. Lemma 7.4 then says that a neighborhood of $x$ must be conformally flat. This holds for every $x \in U$, thus $U$ itself is conformally flat. Thanks to the remark 4.1, $\mathfrak{h}$ is locally conjugated by a smooth conformal diffeomorphism to its holonomy algebra $\mathfrak{h}_{h}$. Proposition 4.8 ensures that the field $X$, hence the algebra $\mathfrak{h}$, is essential on any neighborhood of $x_{0}$. Once again, we are in case (2) of 
theorem 7.1 .

Aknowledgment : I would like to thank warmly Karin Melnick for very interesting conversations on the topic. This work was supported by the ANR GEODYCOS.

\section{REFERENCES}

[A] D.V Alekseevskii: Groups of conformal transformations of Riemannian spaces. Math. USSR, Sb. 18 (1972), 285-301.

[BCH] M.S Capocci, R. Beig, G. S. Hall: Zeros of conformal vector fields, Classical Quantum Gravity 14 (1997), no. 3, 49-52.

[B] A.Besse - Einstein manifolds. Ergebnisse der Mathematik und ihrer Grenzgebiete (3), 10. Springer-Verlag, Berlin, 1987.

[BCDGM] T. Barbot, V. Charette, T. Drumm, W.M. Goldman, K. Melnick: A primer on the $(2+1)$ Einstein universe. Recent Developments in Pseudo-Riemannian Geometry. ESI Lectures in Mathematics and Physics. 2008.

[BFM] U. Bader, C. Frances, K. Melnick: An embedding theorem for automorphism groups of Cartan geometries, Geom. and Func. Analysis 19 (2009), No 2, 333-355.

[BMO] F. Belgun, A. Moroianu, L. Ornea: Essential points of conformal vector fields. eprint arXiv:1002.0482v2.

[CSZ] A. Čap, J. Slovák, V. Žádník: On distinguished curves in parabolic geometries. Transform. Groups 9 (2004), no. 2, 143-166.

[Ca1] M. S. Capocci: Essential conformal vector fields, Classical Quantum Gravity 16 (1999), no. 3, 927-935.

[Ca2] M. S. Capocci: Conformal vector fields and non-degenerate distributions, Classical Quantum Gravity 13 (1996), 1717-1726.

[F] J. Ferrand: The action of conformal transformations on a Riemannian manifold, Math. Ann. 304 (1996), no. 2, 277-291.

[F2] J. Ferrand Les géodésiques des structures conformes. (French) [The geodesics of conformal structures] C. R. Acad. Sci. Paris Sr. I Math. 294 (1982), no. 18, 629-632.

[F3] J. Ferrand: Sur un lemme d'Alekseevskii relatif aux transformations conformes. C. R. Acad. Sci., Paris, Sér. A 284, 121-123 (1977).

[Fr1] C. Frances: Causal conformal vector fields, and singularities of twistor spinors, Ann. Global Anal. Geom. 32 (2007), no. 3, 277-295.

[Fr2] C. Frances: Sur le groupe d'automorphismes des géométries paraboliques de rang 1, Ann. Sci. cole Norm. Sup. (4) 40 (2007), no. 5, 741-764.

English version: eprint arXiv:math/0608.537v1.

[Fr3] C. Frances: Géométrie et dynamique lorentziennes conformes. Thèse, ENS Lyon (2002). Available at http://www.math.u-psud.fr/ frances/.

[Fr4] C. Frances: Dégénérescence locale des transformations conformes pseudoriemanniennes. Preprint. Available at http://www.math.u-psud.fr/ frances/. 
[FM1] C. Frances, K. Melnick: Conformal actions of nilpotent groups on pseudoRiemannian manifolds, Duke Math. J. Volume 153, No 3 (2010), 511-550.

[FM2] C. Frances, K. Melnick: Formes normales pour les champs conformes pseudoriemanniens. in preparation.

[Ko] S. Kobayashi: Transformation groups in differential geometry. Reprint of the 1972 edition. Classics in Mathematics. Springer-Verlag, Berlin, 1995.

[KR1] W. Kühnel, H. B. Rademacher: Essential conformal fields in pseudo-Riemannian geometry. II, J. Math. Sci. Univ. Tokyo 4 (1997), no. 3, 649-662.

[KR2] W. Kühnel, H. B. Rademacher: Essential conformal fields in pseudo-Riemannian geometry, J. Math. Pures Appl. (9) 74 (1995), no. 5, 453-481.

[KR3] W. Kühnel, H.B. Rademacher: Conformal vector fields on pseudo-Riemannian spaces. Differential Geom. Appl. 7 (1997), no. 3, 237-250.

[KR4] W. Kuehnel, H.B. Rademacher: Liouville's theorem in conformal geometry, J. Math. Pures et Appl. (9) (to appear)

[Ob] M. Obata: The conjectures on conformal transformations of Riemannian manifolds. J. Differential Geometry 6 (1971/72), 247-258.

[Sch] R. Schoen: On the conformal and CR automorphism groups, Geom. Funct. Anal. 5 (1995), no. 2, 464-481.

[S] M. Schottenloher: A Mathematical Introduction to Conformal Field Theory, Springer, Berlin, 1997.

[Sh] R.W. Sharpe: Differential Geometry, Cartan's generalization of Klein's Erlangen Program, New York, Springer, 1997.

[St] M. Steller: Conformal vector fields on spacetimes, Ann. Global Anal. Geom. 29 (2006), no. 4, 293-317.

Charles Frances. Laboratoire de Mathématiques, Université Paris-Sud. 91405 ORSAY CEDEX.

E-mail address: Charles.Frances@math.u-psud.fr 